\title{
Proof-of-concept study: profile of circulating microRNAs in Bovine serum harvested during acute and persistent FMDV infection
}

Carolina Stenfeldt, Jonathan Arzt, George Smoliga, Michael LaRocco, Joseph Gutkoska and Paul Lawrence*

\begin{abstract}
Background: Changes in the levels of circulating microRNAs (miRNAs) in the serum of humans and animals have been detected as a result of infection with a variety of viruses. However, to date, such a miRNA profiling study has not been conducted for foot-and-mouth disease virus (FMDV) infection.

Methods: The relative abundance of 169 miRNAs was measured in bovine serum collected at three different phases of FMDV infection in a proof-of-concept study using miRNA PCR array plates.

Results: Alterations in specific miRNA levels were detected in serum during acute, persistent, and convalescent phases of FMDV infection. Subclinical FMDV persistence produced a circulating miRNA profile distinct from cattle that had cleared infection. bta-miR-17-5p was highest expressed during acute infection, whereas bta-miR-31 was the highest during FMDV persistence. Interestingly, miR-1281was significantly down-regulated during both acute and persistent infection. Cattle that cleared infection resembled the baseline profile, adding support to applying serum miRNA profiling for identification of sub-clinically infected FMDV carriers. Significantly regulated miRNAs during acute or persistent infection were associated with cellular proliferation, apoptosis, modulation of the immune response, and lipid metabolism.

Conclusions: These findings suggest a role for non-coding regulatory RNAs in FMDV infection of cattle. Future studies will delineate the individual contributions of the reported miRNAs to FMDV replication, determine if this miRNA signature is applicable across all FMDV serotypes, and may facilitate development of novel diagnostic applications.
\end{abstract}

Keywords: microRNA (miRNA), Foot-and-mouth disease virus (FMDV), Bovine miRNA profile, Serum, Persistence

\section{Background}

Foot-and-mouth disease virus (FMDV) is the etiological agent for foot-and-mouth disease (FMD); one of the most contagious animal diseases known. FMD targets both domestic and wild cloven-hoofed animals, and outbreaks affecting important livestock populations, such as cattle, sheep, and pigs incur enormous economic, political, and social ramifications. Traditional containment strategies combine mass vaccination with large scale animal culling. FMDV is represented globally by seven

\footnotetext{
* Correspondence: paul.lawrence@biocogent.com

Plum Island Animal Disease Center, USDA/ARS/NAA/FADRU, P.O. Box 848, Greenport, NY 11944-0848, USA
}

different serotypes: O, A, C, Asia1, SAT1, SAT2, and SAT3; and within each serotype there are multiple subtypes and variants. The virus is the prototypic member of the Aphthovirus genus of the family Picornaviridae and possesses a single-stranded positive-sense RNA genome of approximately 8500 nucleotides (nts), which consists of a large open reading frame (ORF) flanked by 5 , and 3' non-translated regions (NTRs). The positivesense RNA functions as a messenger RNA (mRNA) from which a single polyprotein is translated and subsequently proteolyzed into a series of intermediate and mature viral proteins. The ORF is traditionally sub-divided into three regions: P1, P2, and P3. The P1 region encodes 
four structural proteins, which assemble into the FMDV capsid, whereas the P2 and P3 regions encode the virus non-structural proteins essential for virus replication. The 5' and 3' NTRs flanking the ORF exhibit multiple secondary RNA structures, which regulate the stepwise translation of virus proteins and replication of the RNA genome. Knowledge obtained from the study of the molecular biology and pathogenesis of FMDV continues to be applied to the development of improved vaccines and anti-viral therapies [1,2].

RNA interference (RNAi), a process by which mRNA transcripts are targeted for degradation or translational repression by short non-coding RNA molecules (ncRNAs) has been widely studied for its utility as an anti-viral therapeutic platform. Small interfering RNAs (siRNAs) are generated when large double-stranded RNA (dsRNA) molecules are gradually processed into progressively smaller dsRNA of approximately 20-22 nucleotides in length; and have been investigated as anti-viral therapeutics [3-5]. In the case of FMDV, siRNAs targeted to sequences in the virus genome have been tested for their capacity to impede the progression of viral infection with reportedly mixed benefits [6-9]. A key drawback of siRNA-based anti-viral therapies is the required complete sequence complementarity between the siRNA and its cognate RNA target. RNA viruses are highly mutable, and as such, the accumulation of mutations with each replication cycle diminishes the efficacy of the siRNA treatment. Additionally, with seven different serotypes and multiple subtypes and variants of FMDV, designing siRNAs that are universally functional across virus strains would be highly challenging.

In contrast to siRNAs, microRNAs (miRNAs) do not require perfect complementarity to their target RNA molecule [10-13], which mitigates the effect of mutations in the RNA genome and facilitates a similar function across multiple forms of FMDV. Another key difference between siRNAs and miRNAs is that siRNAs are generally produced from exogenous RNA molecules, while miRNAs are generated from endogenous RNA molecules [12]. In the normal cellular environment, miRNAs act as key posttranscriptional regulators of gene expression for a variety of biological processes including immune response, cell proliferation, and metabolism [14-16].

Currently, miRNAs are also being explored as potential bio-markers of infectious disease, particularly miRNAs that are detectable in the bloodstream [17-21]. Studies have shown that different viruses generate distinct expression profiles of circulating miRNA, so much so that one group was able to distinguish between enterovirus 71 and coxsackievirus 16 as the etiological agents for individual cases of hand-foot-and-mouth disease [17]. Unfortunately, while greater than 2588 human miRNAs have been identified, only approximately 798 bovine miRNAs have been fully characterized (release 21, June 2014, miRBase.org). Furthermore, while the miRNA response to several bacterial pathogens has been examined in cattle, very few studies have been conducted in the context of viral infection [22].

Although still an evolving technology, the information gained from miRNA profiling investigations has contributed substantially to efforts for curtailing viral infection. One strategy has been to incorporate miRNA target sequences into viral genomes that target host anti-viral response pathways to contribute to the development of live attenuated vaccine platforms, which has been accomplished for vesicular stomatitis virus [23], Japanese encephalitis virus [24], coxsackievirus A21 [23], and influenza virus $[25,26]$. Contrastingly, some viruses have exploited the opposite approach, as several DNA viruses and a few RNA viruses have been identified that harbor functional miRNA sequences [27-29]. Of relevance to FMDV, miRNA sequences were identified in the antigenome (or negative strand intermediate) of a representative Picornavirus, hepatitis A virus (HAV), thus demonstrating that RNA viruses, even those whose replication cycle is confined to the cytoplasm, can encode functional miRNAs like their DNA virus counterparts $[28,29]$. Although certainly still controversial, these findings contest the notion that RNA viruses would not encode functional miRNAs since interactions with the cellular miRNA machinery would require: (i) localization of an RNA virus genome to the nucleus and (ii) that processing of the pre-miRNA hairpin would irreparably damage the virus genome.

An alternative anti-viral miRNA strategy has been to introduce artificial miRNAs in cell culture or in infected animals to target specific viral sequences or the mRNA transcripts of cellular proteins that the virus requires for an efficient replication cycle. This approach has been beneficially used in two reported instances involving FMDV, where artificial miRNAs were constructed that targeted either the IRES sequence in the 5' NTR or the coding sequence for the $3 \mathrm{D}$ polymerase [30, 31]. When these constructs were transfected into cell culture prior to FMDV infection, replication was significantly impeded. In another study, the artificial miRNA sequences were inserted into the genomic DNA of a cell line, so as to allow for constitutive expression of the anti-FMDV miRNAs [32]. However, this failed to protect the cells from FMDV infection due to inefficient localization of the anti-FMDV miRNAs to sites of virus replication in the sub-cellular environment.

This current work comprises a proof-of-concept study in which the differential regulation of serum miRNAs was investigated in cattle infected with FMDV serotype A isolate A24 Cruzeiro. The bovine serum miRNA profile was characterized using quantitative reverse 
transcriptase polymerase chain reaction (RT-qPCR) array plates designed to detect the relative abundance levels of 169 of the bovine miRNAs characterized in the past several years (Table 1, Fig. 1c). Serum from uninfected animals was compared to samples obtained through three distinct phases of FMDV infection: acute infection (peak viremia), persistent infection (subclinical persistence of FMDV in the upper respiratory tract), and convalescent phase, comprising animals that had successfully cleared the infection (Fig. 1a, b). Distinct miRNA signatures of up- and down-regulated miRNAs in circulation in response to FMDV infection were detected. The results obtained from this proof-of-concept study reinforce the application of this approach to future large scale testing and the investigation of miRNA signatures for other serotypes of FMDV.

\section{Methods}

\section{Animals}

Serum samples used for this investigation were derived from non-vaccinated animals included in studies investigating the pathogenesis of acute and persistent phases of FMDV infection in cattle [33, 34]. The cattle used were $\sim 200 \mathrm{~kg}(\mathrm{~kg})$ Holstein cattle that were 6-8 months old, and were purchased from Thomas D. Morris Inc. (Reisterstown, MD). Briefly, cattle were infected with FMDV serotype A isolate A24 Cruzeiro via intranasopharyngeal (INP) inoculation [35] at a dose of $10^{5}$ BTID $_{50}(50 \%$ infectious doses titrated using bovine tongue). The INP inoculation method represents a needlefree "simulated-natural system", where a volume of inoculum is introduced to the nasopharynx of cattle via a plastic catheter that has been calibrated for optimal deposition.

Table 1 Bovine miRNA array

\begin{tabular}{|c|c|c|c|c|c|}
\hline \multicolumn{6}{|l|}{ miRNA } \\
\hline bta-miR-1 & bta-miR-30a-5p & bta-miR-127 & bta-miR-150 & bta-miR-222 & bta-miR-1179 \\
\hline bta-miR-7 & bta-miR-30b-5p & bta-miR-128 & bta-miR-151-3p & bta-miR-320a & bta-miR-1185 \\
\hline bta-miR-10a & bta-miR-30c & bta-miR-129-3p & bta-miR-151-5p & bta-miR-331-3p & bta-miR-1193 \\
\hline bta-miR-10b & bta-miR-30d & bta-miR-129-5p & bta-miR-152 & bta-miR-342 & bta-miR-1197 \\
\hline bta-miR-15a & bta-miR-30e-5p & bta-miR-130a & bta-miR-153 & bta-miR-345-5p & bta-miR-1224 \\
\hline bta-miR-15b & bta-miR-31 & bta-miR-130b & bta-miR-154a & bta-miR-361 & bta-miR-1225-3p \\
\hline bta-miR-16b & bta-miR-34a & bta-miR-132 & bta-miR-181a & bta-miR-363 & bta-miR-1248 \\
\hline bta-miR-17-3p & bta-miR-34b & bta-miR-133a & bta-miR-181b & bta-miR-365-3p & bta-miR-1249 \\
\hline bta-miR-17-5p & bta-miR-34c & bta-miR-133b & bta-miR-181c & bta-miR-369-3p & bta-miR-1256 \\
\hline bta-miR-18a & bta-miR-93 & bta-miR-134 & bta-miR-186 & bta-miR-369-5p & bta-miR-1271 \\
\hline bta-miR-18b & bta-miR-98 & bta-miR-135a & bta-miR-191 & bta-miR-374a & bta-miR-1281 \\
\hline bta-miR-19a & bta-miR-99a-5p & bta-miR-135b & bta-miR-192 & bta-miR-380-3p & bta-miR-1282 \\
\hline bta-miR-19b & bta-miR-99b & bta-miR-136 & bta-miR-193a-3p & bta-miR-380-5p & bta-miR-1287 \\
\hline bta-miR-20a & bta-miR-100 & bta-miR-137 & bta-miR-193a-5p & bta-miR-423-3p & bta-miR-1291 \\
\hline bta-miR-20b & bta-miR-101 & bta-miR-138 & bta-miR-195 & bta-miR-425-3p & bta-miR-1296 \\
\hline bta-miR-21-3p & bta-miR-103 & bta-miR-139 & bta-miR-199a-3p & bta-miR-425-5p & bta-miR-1298 \\
\hline bta-miR-21-5p & bta-miR-105a & bta-miR-140 & bta-miR-199a-5p & bta-miR-450a & bta-miR-1301 \\
\hline bta-miR-22-5p & bta-miR-105b & bta-miR-141 & bta-miR-199b & bta-miR-455-3p & bta-miR-1306 \\
\hline bta-miR-23a & bta-miR-106a & bta-miR-142-3p & bta-miR-200a & bta-miR-455-5p & bta-miR-1307 \\
\hline bta-miR-23b-3p & bta-miR-106b & bta-miR-142-5p & bta-miR-200b & bta-miR-484 & bta-let-7a-3p \\
\hline bta-miR-24-3p & bta-miR-107 & bta-miR-143 & bta-miR-200c & bta-miR-487a & bta-let-7a-5p \\
\hline bta-miR-25 & bta-miR-122 & bta-miR-144 & bta-miR-204 & bta-miR-487b & bta-let-7b \\
\hline bta-miR-26a & bta-miR-124a & bta-miR-145 & bta-miR-205 & bta-miR-497 & bta-let-7c \\
\hline bta-miR-26b & bta-miR-124b & bta-miR-146a & bta-miR-210 & bta-miR-499 & bta-let-7d \\
\hline bta-miR-27a-3p & bta-miR-125a & bta-miR-146b & bta-miR-214 & bta-miR-532 & bta-let-7e \\
\hline bta-miR-27b & bta-miR-125b & bta-miR-147 & bta-miR-215 & bta-miR-545-3p & bta-let-7f \\
\hline bta-miR-29a & bta-miR-126-3p & bta-miR-148a & bta-miR-218 & bta-miR-545-5p & bta-let-7 g \\
\hline bta-miR-29b & bta-miR-126-5p & bta-miR-148b & bta-miR-221 & bta-miR-660 & bta-let-7i \\
\hline bta-miR-29c & & & & & \\
\hline
\end{tabular}

Panel of 169 established bovine miRNAs that were profiled for differential expression in response to FMDV infection in cattle serum 


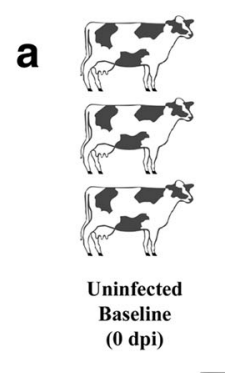

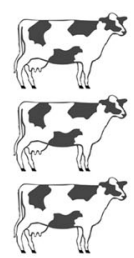

Viremic Acutely Infected (3 dpi)

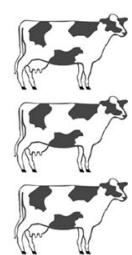

Non-Carrier

Cleared (35 dpi)

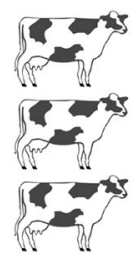

Carrier

Persistently Infected

(35 dpi)

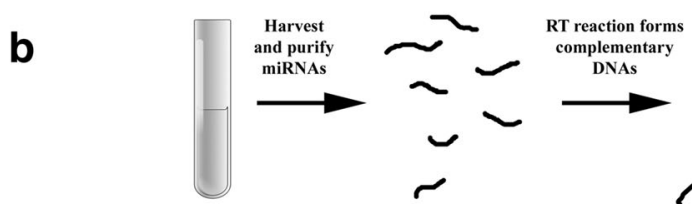

Serum

miRNAs
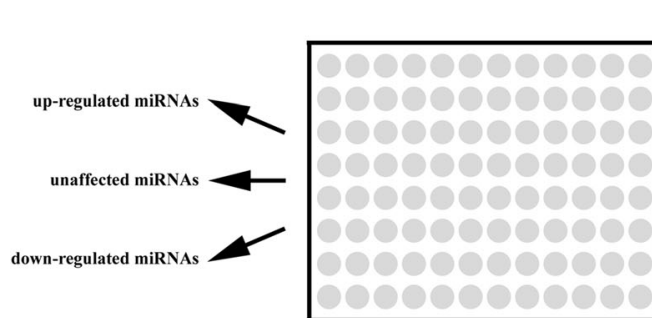

RT-PCR analysis

on miRNA primer

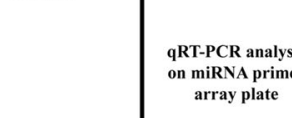

C

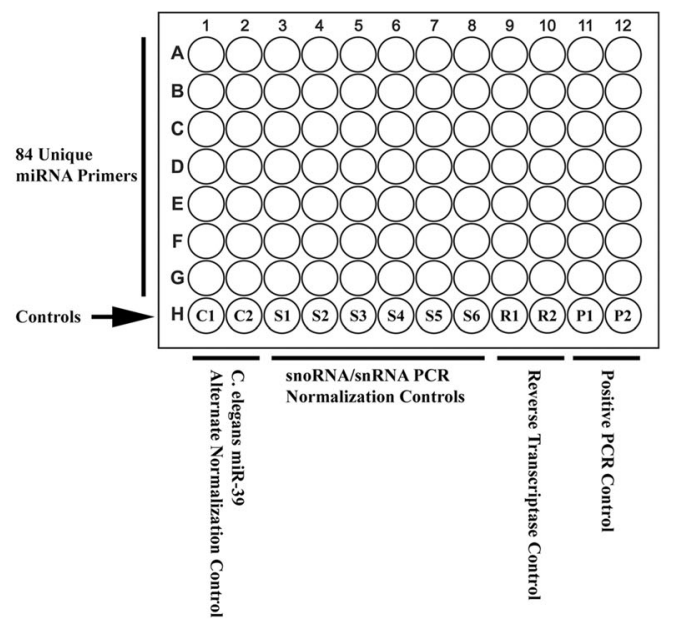

Fig. 1 Schematic overview of miRNA profiling study. a Serum was collected from three different groups of FMDV-infected cattle: acutely infected (viremic; 3-4 dpi), persistently infected ("FMDV carriers"; 35 dpi) and convalescent ("non-carriers"; 35 dpi), and were compared to uninfected controls. Each group comprised serum samples from three animals. b miRNAs were purified from individual serum samples. The purified miRNAs were reverse transcribed into complementary DNA (CDNA). The CDNA samples were then analyzed by RT-PCR on bovine miRNome miRNA array plates containing primers to 169 different bovine miRNAs. The results obtained indicated which miRNAs were left unchanged, up-regulated, or down-regulated in response to FMDV infection. c schematic of the organization of the miRNA PCR array plates utilized in this study

The procedures employed for this study were approved by the Plum Island Animal Disease Center (PIADC) Institutional Animal Care and Use Committee (IACUC) under protocols: 209-12-R and 209-15-R.

\section{Serum sample collection}

Whole blood was collected in serum separation tubes, which were stored on ice until downstream centrifugation in the laboratory for harvesting the serum. The serum was then stored at $-70^{\circ} \mathrm{C}$ until ready for subsequent laboratory testing. Serum samples for miRNA profiling came from whole blood harvested during acute infection, which was defined as the day on which peak viremia occurred (3 or 4 days post-inoculation [dpi]; data not shown). Additional samples were obtained during the persistent phase of infection ( $35 \mathrm{dpi})$ from cattle that were defined as either persistently infected "FMDV carriers", or from animals that had successfully 
cleared the infection; "non-carriers"/convalescent animals. The distinction between FMDV carriers and noncarriers was made based on detection of FMDV in oropharyngeal fluids beyond 28 dpi (data not shown) [34]. Serum collected from cattle prior to inoculation with FMDV was used as a baseline for comparison with infected and convalescent animals. Each test group comprised sera from three individual animals. Samples used in this study are listed in Additional file 1: Table S3.

\section{miRNA isolation}

miRNAs were harvested from serum samples using the miRNeasy serum/plasma kit (Qiagen, catalog \# 217184) following manufacturer's instructions. The miRNA harvesting process included the addition of the miRNeasy serum/plasma spike-in control (Qiagen, catalog \# 219610) to facilitate downstream quality control. Extracted miRNA was immediately used for cDNA synthesis.

\section{cDNA synthesis}

cDNA was generated from purified miRNA using the miScript II RT (reverse transcriptase) kit purchased from Qiagen (Catalog \# 218160) following manufacturer's instructions. Reaction mixes were incubated at $37^{\circ} \mathrm{C}$ for $60 \mathrm{~min}$, followed by $5 \mathrm{~min}$ at $95^{\circ} \mathrm{C}$, and finally cooled to $4{ }^{\circ} \mathrm{C}$. The cDNA samples were then stored at $-70^{\circ} \mathrm{C}$ until subsequent analysis.

miScript miRNA PCR array cow miRNome ${ }^{\mathrm{Tm}}$ profiler plates Ninety-six well miRNA PCR array plates for the bovine miRNome $^{\mathrm{rm}}$ were purchased from Qiagen (Catalog \# 331221, Product \# MIBT-651Z and MIBT-652Z). In addition to wells containing primers for the miRNA species listed in Table 1 and shown in Fig. 1, both array plates possessed wells containing C. elegans miR-39, snoRNA, and snRNA for normalization of the data as well as a reverse transcription control (miRTC) and positive control for the PCR reaction (PPC).

\section{Reverse transcription quantitative PCR (RT-qPCR)}

The cDNA generated in the prior RT step was used as template material for the downstream RT-qPCR analysis. The cDNA template was diluted and mixed with the reagents provided in the miScript SYBR Green PCR kit purchased from Qiagen (Catalog \# 218076), consisting of QuantiTect SYBR green master mix and miScript universal primer. $25 \mu \mathrm{L}$ of combined template and reagents was loaded onto Qiagen bovine miRnome ${ }^{\mathrm{mm}}$ miScript miRNA PCR array plates 1 and 2 (Product \# MIBT$651 \mathrm{Z}$ and MIBT-652Z). The PCR array plates were analyzed on ABI 7500 thermal cyclers (Applied Biosystems, Life Technologies, Foster City, CA) using the following cycling parameters: an initial activation of the HotStar ${ }^{\mathrm{Tx}}$
Taq DNA polymerase at $95^{\circ} \mathrm{C}$ for $15 \mathrm{~min}$, followed by 40 iterative cycles of $15 \mathrm{~s}(\mathrm{sec})$ at $94{ }^{\circ} \mathrm{C}, 30 \mathrm{~s}$ at $55^{\circ} \mathrm{C}$, and $30 \mathrm{~s}$ at $70^{\circ} \mathrm{C}$.

\section{miRNA expression profile analysis}

The $\mathrm{C}_{\mathrm{t}}$-values accumulated from the RT-PCR analyses were analyzed using the Qiagen GeneGlobe (http:// www.qiagen.com/us/shop/genes-and-pathways/data-anal ysis-center-overview-page/mirna-pcr-array-mirbase-prof iler-plates-data-analysis-center/) online software plat form (formerly SABiosciences: pcrdataanalysis.sabioscie nces.com/mirna/arrayanalysis.php). The quality of the miRNA extractions, RT reactions and PCR efficiency were evaluated against internal controls included on each PCR array plate. The data was normalized using the "global $C_{t}$ mean of expressed miRNAs" method provided in the data analysis platform. Post-normalization of the $C_{t}$ values, the fold changes in miRNA expression in each infected and convalescent sample relative to control samples was calculated using the $\Delta \Delta \mathrm{C}_{\mathrm{t}}$ method [36]. The significance of the fold-changes was evaluated using two-tailed t-tests comparing each test group (acute, FMDV carrier, and non-carrier) to the uninfected control group. Significant levels were set at fold change $>1.50$ and $p$-value $<0.05$.

\section{Cell culture}

LFBK- $\alpha v \beta 6$ cells $[37,38]$ were generously provided by Dr. Luis Rodriguez (PIADC, ARS, USDA). The cells were cultured in Dulbecco's minimal essential medium (DMEM) supplemented with $10 \%$ fetal bovine serum (FBS) and $1 \%$ antibiotic/antimycotic (A/A) at $37^{\circ} \mathrm{C}$ in humidified incubator with $5 \% \mathrm{CO}_{2}$.

\section{miRNA mimic transfection and infection}

LFBK- $\alpha v \beta 6$ cells were grown to approximately $30-40 \%$ confluence and transfected with mimics to miR-17-5p, miR-1281 (Thermo Scientific, Waltham, MA), a nonsense negative control miR (miR-NC), a control miR342-5p that does not impact FMDV infection, or left untransfected following the RNAiMAX (Thermo Scientific, Waltham, MA) transfection method (manufacturer's protocol). Forty-eight hours post-transfection, the transfected cells were inoculated with FMDV A24 Cruzeiro at a multiplicity of infection (MOI) of $10^{-3}$ and allowed to incubate for $24 \mathrm{~h}$. The resulting virus titers were then determined for the collected samples following a previously described protocol [39]. Briefly, each sample was diluted $10^{-1}$ to $10^{-6}$ in virus growth medium (1\% FBS, 1\% A/A, and $25 \mathrm{mM}$ HEPES in DMEM) and the dilutions were applied to LFBK- $\alpha \mathrm{v} \beta 6$ cell monolayers, incubated for $1 \mathrm{~h}$ at $37^{\circ} \mathrm{C}$, acid-washed and rinsed, following by application of gum tragacanth overlay. The plates were then incubated overnight at $37^{\circ} \mathrm{C}$, 
after which they were fixed and stained with crystal violent in Histochoice fixative (Amersco, Solon, $\mathrm{OH}$ ). The plaques were counted and the calculated plaque forming units (PFU) per milliliter were plotted using Microsoft Excel (Microsoft Corporation, Redmond, WA).

\section{Results \\ Differential regulation of miRNAs in serum during acute FMDV infection}

To determine if FMDV infection of cattle (both acute and persistent) would induce an alteration in the abundance of circulating miRNAs in the serum, cattle were inoculated in triplicate with FMDV A24 Cruzeiro via the intra-nasopharyngeal (INP) route [40]. Serum was collected in acutely infected cattle at $3 \mathrm{dpi}$ (viremic) as well as at $35 \mathrm{dpi}$ in cattle that either cleared the virus (noncarriers) or became persistently infected with FMDV (carriers) (Fig. 1a). Serum was also collected from cattle prior to inoculation with FMDV as a baseline for comparison with infected animals. RNA was harvested and miRNA purified from the samples from which complementary DNA (cDNA) was generated via reverse transcriptase reaction (Fig. 1b). The resulting cDNAs were used as templates for RT-qPCR reactions on miRNA PCR array plates (Qiagen miScript Bovine miRNome) containing a series of primers unique to 169 characterized bovine miRNAs (Table 1) so as to identify miRNAs showing either up-regulation or down-regulation in the collected serum samples (Fig. 1c). The triplicate samples from each experimental set (acute, non-carrier, and carrier) were compared against uninfected controls, and first evaluated for quality of RT reaction and PCR efficiency; for which passing samples were then normalized against internal standards. Post-normalization, fold changes in miRNA levels were only accepted that exhibited statistical significance with $p$-values $<0.05$ across triplicate samples, and were only reported if a greater than 1.50-fold change was detected.

Importantly, to avoid artifacts of technique, there was no pre-amplification of miRNAs prior to evaluation on the miRNA PCR arrays, which can potentially artificially increase levels of background miRNAs. By this approach, 7 bovine miRNAs were found up-regulated in serum collected from cattle acutely infected with FMDV (3-4 dpi) relative to uninfected controls. Raw scores are shown in Table 2, while volcano plots of $\log 2$ fold changes in miRNA levels are shown in Fig. 2a. The upregulated miRNA species included bta-miR-17-5p, btamiR-146a, bta-miR-144, bta-miR-34a, bta-miR-369-3p, bta-miR-497, and bta-miR-22-5p (Table 2 and Fig. 2a). The three most up-regulated miRNAs were bta-miR-17$5 p(+35.88$ fold increase), bta-miR-146a $(+34.36$ fold increase), and bta-miR-144 (+28.78 fold increase) (Table 2). In contrast, three of the measured targets were significantly down-regulated during acute FMDV infection: bta-let-7 g (-1.96 fold decrease), bta-miR-1281 ( -2.50 fold decrease), and bta-miR-26b (-3.09 fold decrease) (Table 2 and Fig. 2a). Some of the reported functionalities of these miRNA species are detailed in Table 2 to extrapolate for possible roles in acute FMDV infection.

\section{Differential regulation of miRNAs in serum during persistent FMDV infection}

The FMDV carrier state is defined by continuous shedding of low quantities of infectious virus in oropharyngeal fluids beyond $28 \mathrm{dpi}[41,42]$. This persistent phase of FMDV infection occurs in approximately $50-100 \%$ of FMDV-infected cattle, and is characterized by subclinical presence of actively replicating FMDV within the nasopharyngeal mucosa $[34,43,44]$. In contrast to acute FMDV infection, the persistent phase of infection is not associated with a marked local inflammatory reaction or a detectable systemic anti-viral response [34, 45]. Nine of the measured miRNAs were significantly up-regulated in persistently infected FMDV carriers compared to uninfected controls; and once again, raw scores are shown in Table 3, while volcano plots of $\log 2$ fold changes in miRNA levels are shown in Fig. 2c. As shown in the top portion of Table 3: bta-miR-22-5p, bta-miR-147, btamiR-1224, bta-miR-144, bta-miR-497, bta-miR-154a, bta-miR-17-5p, bta-miR-205, and bta-miR-31, with fold changes of $2.17,5.28,5.69,23.78,24.62,24.05,40.84$, 41.22 , and 43.37 , respectively. The corresponding volcano plots for the two array plates are shown in Fig. 2c. Bta-miR-205 was the strongest induced miRNA during persistent infection. Three miRNAs were significantly down-regulated in serum from persistently infected cattle: bta-miR-1281, bta-miR-181b, and bta-miR-23b-5p with fold decreases of $3.41,2.77$, and 2.44, respectively (Table 3). The strongest down-regulated miRNA, miR1281, was also observed to have decreased levels in the serum collected from acutely infected cattle.

As could be expected, the miRNA expression profile in cattle that had successfully cleared FMDV infection was more similar to that of the uninfected controls. In this cohort of animals, only two miRNAs were differentially expressed compared to the baseline: bta-miR- 150 and bta-miR-455-3p, which were down-regulated 1.65 and up-regulated 68.17 fold, respectively (Table 3). Notably, bta-miR-455-3p was the highest up-regulated miRNA detected in the entire proof-of-concept study; see outlying miRNA in the far right of the volcano plot for array plate 2 (Fig. 2b). The non-carrier results reinforce the viability of this miRNA profiling approach described in this proof-of-concept study given that virtually all of the miRNA species have returned to baseline levels. As mentioned in the previous section, some of 
Table 2 Bovine miRNAs up-regulated and down-regulated in cow serum in response to acute FMDV infection

\begin{tabular}{|c|c|c|c|c|}
\hline \multicolumn{5}{|c|}{ miRNA regulation in Bovine serum during acute FMDV infection } \\
\hline miRNA & Fold change & $p$-value & Ascribed function & Ref \\
\hline miR-26b & -3.09 & 0.032 & $\begin{array}{l}\square \text { Role in adipogenesis and adipocyte differentiation } \\
\square \text { Inhibits HBV replication by targeting CHORDC1 } \\
\square \text { Inhibits HCV replication in PBMCs } \\
\square \text { Promotes RSV replication by targeting TLR4 } \\
\square \text { Tumor suppressor: targets USP9X, PTGS2, CDK8, TAK1, and TAB3 }\end{array}$ & $\square[69-76]$ \\
\hline miR-1281 & -2.50 & 0.020 & $\square$ Lipid metabolism: in dairy cattle, role in adipogenesis & $\square[77]$ \\
\hline let-7 g & -1.96 & 0.024 & $\begin{array}{l}\square \text { Cellular proliferation: targets lectin-like oxidized low density lipoprotein receptor-1 } \\
(\text { LOX), caspase-3, and Aurora-B }\end{array}$ & $\square[78-80]$ \\
\hline miR-22-5p & +2.73 & 0.030 & $\square$ Tumor suppressor: targets CDK6, SIRT1, and Sp1 & $\square[81-88]$ \\
\hline miR-497 & +26.16 & 0.031 & $\begin{array}{l}\text { 口 Tumor suppressor: targets CCNE1, insulin-like growth factor } 1 \text { receptor (IGF-1R), } \\
\text { checkpoint kinase } 1(C H K 1) \text {, represses elF4E, E3 ubiquitin ligase }\end{array}$ & ๑ [89-93] \\
\hline miR-369-3p & +28.57 & 0.015 & $\begin{array}{l}\square \text { Cellular proliferation: targets N-cadherin } \\
\square \text { Down-regulates adipogenic differentiation in mesenchymal stromal cells }\end{array}$ & $\square[94,95]$ \\
\hline miR-34a & +28.57 & 0.015 & $\begin{array}{l}\square \text { Tumor suppressor: targets CCND1, CDK6, FMNL2 and E2F5; activated by p53 } \\
\square \text { Regulates lipid metabolism with HNF4a } \\
\square \text { Immune modulatory: targets IL-6 and IL-8 } \\
\square \text { Found in bovine milk }\end{array}$ & $\square$ [96-101] \\
\hline miR-144 & +28.78 & 0.015 & $\begin{array}{l}\text { - Tumor suppressor: targets CCNE1, ROCK1, ROCK2, EZH2, TIGAR, AKT3, E2F3, } \\
\text { ADAMTS5, ADAM10, RAB14, and C-Met }\end{array}$ & $\square[102-112]$ \\
\hline miR-146a & +34.36 & 0.018 & $\begin{array}{l}\square \text { Tumor suppressor: targets SOS1 } \\
\square \text { Up-regulated by HBV X protein } \\
\square \text { Immune modulatory: induced by NF-KB signaling; targets IRAK1 and TRAF6, thus } \\
\text { diminishing pro-inflammatory response from TLR signaling }\end{array}$ & $\square$ [113-120] \\
\hline miR-17-5p & +35.88 & 0.038 & $\begin{array}{l}\square \text { Cellular proliferation: targets SMAD7, FBXO31, E2F1, and c-Myc } \\
\square \text { Repressed HCV infection, inversely correlated with HCV treatment response } \\
\square \text { HIV-1 Tat C modulates NOX2 and NOX4 via miR-17 } \\
\square \text { Immune modulatory: suppresses TLR signaling through IL6 and represses } \\
\text { IFN-stimulated MXA expression }\end{array}$ & $\square[121,122]$ \\
\hline
\end{tabular}

List of bovine miRNAs that were observed to be up-regulated and down-regulated in serum samples collected during the acute phase of FMDV infection. Shown are the miRNA species, the fold-change in expression levels (in ascending order), and the ascribed function from literature searches of cattle miRNAs and homologous miRNAs from other species with little to no sequence divergence. The miRNAs listed showed a fold-change in expression levels of greater than 1.50 , and had $p$-values of $<0.05$

the reported functionalities associated with these miRNAs is described in Table 3, allowing for hypothetical contributions to be formulated.

\section{Genomic localization of the differentially expressed miRNAs}

To further characterize the miRNAs that were found to be differentially regulated in response to FMDV infection, we sought to determine if there was any correlation between the miRNAs with respect to where their sequences map in the Bos taurus genome. To that end, the miRNAs were queried in the most current version of miRbase (release 21, June 2014, miRBase.org) for their chromosomal sequence sites. As shown in Fig. 3, the encoded sequences of the miRNAs detected in this study were found scattered across bovine chromosomes: 1,2 , $5,7,8,10,11,12,16,18,19,21$, and 22 . The only chromosomes in the Bos taurus genome that were associated with more than one of the identified miRNAs were: chromosome \#8 with bta-miR-23b-5p, bta-miR-31, and bta-miR-455-3p; chromosome \#16 with bta-miR-34a,
bta-miR-181b, and bta-miR-205; chromosome \#19 with bta-miR-22-5p, bta-miR-144, and bta-miR-497; and finally chromosome \#21 with bta-miR-154a and btamiR-369-3p. The miRBase database was also queried for whether the gene sequences for the identified miRNAs were localized to intergenic or intronic regions of the Bos taurus genome. Eleven of the miRNAs are encoded in intergenic regions, including: bta-miR-1281, btamiR-150, bta-miR-181b, bta-miR-497, bta-miR-144, bta-miR-34a, bta-miR-154a, bta-miR-146b, bta-miR17-5p, bta-miR-205, and bta-miR-31. The remaining 8 miRNAs are encoded within intronic regions: btamiR-26b, bta-miR-455-3p, bta-miR-23b-5p, bta-let-7 g, bta-miR-22-5p, bta-miR-147, bta-miR-369-3p, and btamiR-1224. Based on these findings, we concluded that there was no pattern to where in the bovine genome the detected miRNAs were expressed.

\section{Cluster analysis of the differentially expressed miRNAs}

Many miRNA sequences encoded in the genomes of various species have been discovered clustered with 

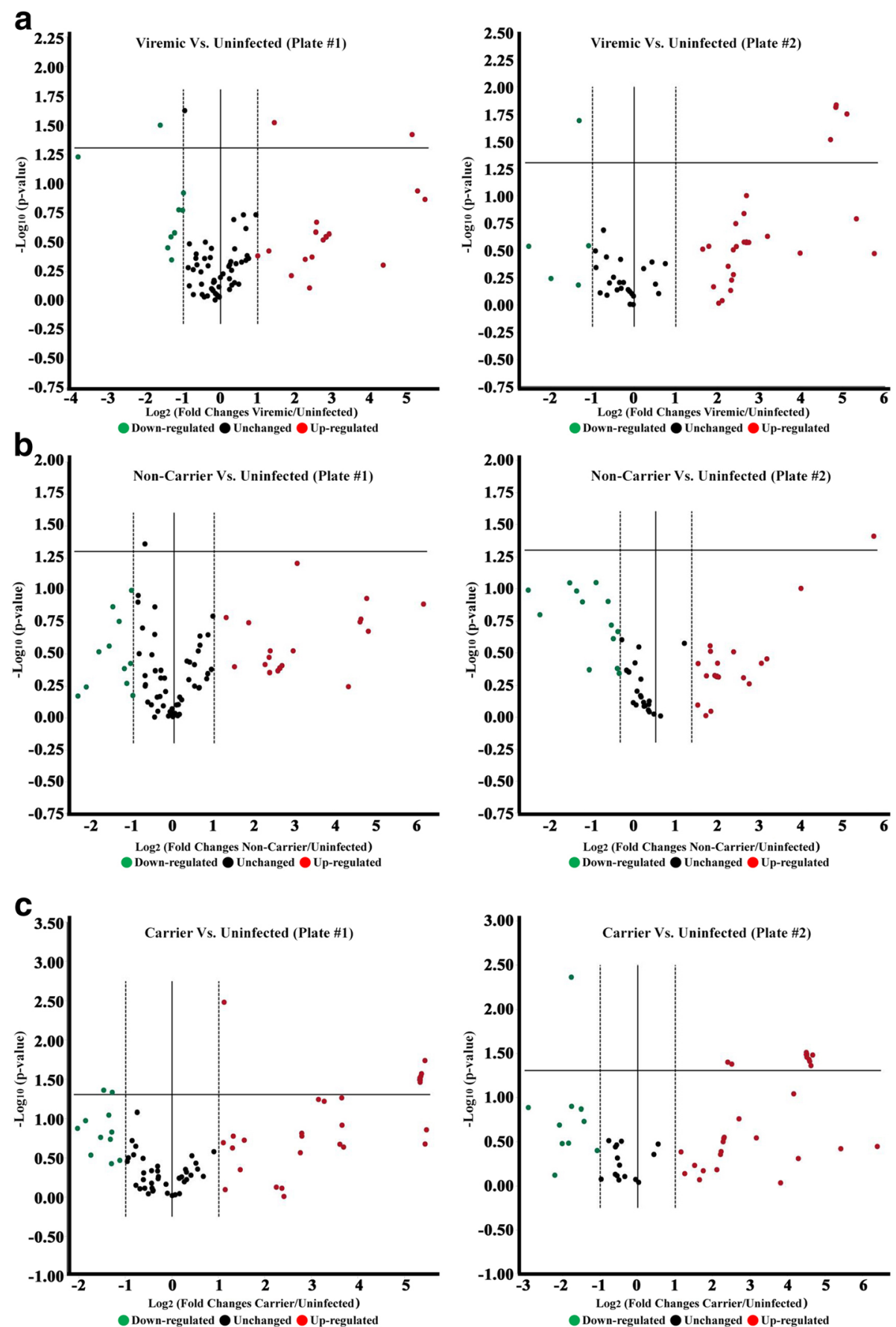

Fig. 2 Differentially expressed miRNAs in response to FMDV infection. The expression patterns of 169 distinct bovine miRNAs was evaluated in serum harvested during three distinct phases of FMDV infection: a acute (viremic) b convalescent "non-carrier" and $\mathbf{c}$ persistently infected "carrier". Expression levels were compared against serum from uninfected controls and are plotted onto volcano plots with the fold change in expression on the $\mathrm{X}$-axis and the degree of reproducibility between replicates ( $\mathrm{p}$-value) on the $\mathrm{Y}$-axis. Circles: miRNAs with unchanged expression, down-regulated expression (green), and up-regulated expression (red). miRNAs with significantly regulated expression (fold-change >1.5 and $p$-value of $<0.05$ [or greater than 1.25 by the $-\log _{10}$ ] are plotted above the horizontal lines indicated on the volcano plots

other miRNAs that often share similar regulatory functions [46, 47]. Given that, cluster analysis was performed on the differentially regulated bovine miRNAs detected in this study using two different sources: miRbase (release 21, June 2014, miRBase.org) and MetaMirClust [48]. These two databases provided corroborating data regarding whether the detected miRNAs were non-clustered or clustered, and what 
Table 3 Bovine miRNAs up-regulated and down-regulated in cow serum in response to persistent FMDV infection

\begin{tabular}{|c|c|c|c|c|}
\hline \multicolumn{5}{|c|}{$\begin{array}{l}\text { miRNA regulation in Bovine serum during persistent FMDV infection } \\
\text { Persistently Infected FMDV Carriers }\end{array}$} \\
\hline miRNA & Fold change & $p$-value & Ascribed function & Ref \\
\hline miR-1281 & -3.41 & 0.004 & $\square$ Lipid metabolism: in dairy cattle, role in adipogenesis & $\square[50]$ \\
\hline miR-181b & -2.77 & 0.044 & $\begin{array}{l}\square \text { Cellular proliferation: targets RASSF1A and NF-KB } \\
\square \text { Immune modulatory: adenylyl cyclase } 9 \text { (AC9), antagonistic to IFNa expression }\end{array}$ & $\square[123-126]$ \\
\hline miR-23b-5p & -2.44 & 0.047 & $\begin{array}{l}\square \text { Tumor suppressor: up-regulated by p53, targets mitochondrial glutaminase, } \\
\text { antagonizes c-Myc signaling, and suppresses metastasis } \\
\square \text { Cellular proliferation: targets proline oxidase }\end{array}$ & $\square[127-132]$ \\
\hline miR-22-5p & +2.17 & 0.003 & $\begin{array}{l}\square \text { Tumor suppressor: targets CDK6, SIRT1, and Sp1 } \\
\square \text { Circulating miRNA bio-marker for Huntington's Disease and acute phase } \\
\text { myocardial infarction } \\
\square \text { Involved in neonatal heart development } \\
\square \text { Anti-inflammatory: negatively regulates type I IFN inflammatory cytokine } \\
\text { response }\end{array}$ & $\square[82-86,88]$ \\
\hline miR-147 & +5.28 & 0.039 & $\begin{array}{l}\square \text { Anti-inflammatory: TLR2, TLR3, and TLR4 stimulate miR-147 expression, } \\
\text { which participates in negative feedback loop to suppress inflammatory } \\
\text { cytokine expression } \\
\square \text { Up-regulated by HCV Genotype-4 } \\
\square \text { Tumor suppressor: targets HOXC6 oncogene }\end{array}$ & $\square$ [133-135] \\
\hline miR-1224 & +5.69 & 0.042 & $\square$ Tumor suppressor: targets CREB1 & $\square[136]$ \\
\hline miR-144 & +23.78 & 0.037 & $\begin{array}{l}\text { - Tumor suppressor: targets cyclin E1 (CCNE1), ROCK1, ROCK2, SMAD4, TIGAR, } \\
\text { E2F3, ADAMTS5, ADAM10, RAB14, ZEB1/2, and c-Met }\end{array}$ & $\square$ [103-112] \\
\hline miR-154a & +24.05 & 0.039 & $\square$ Tumor suppressor: targets E2F5, ZEB2, Wnt5a, Wnt11, TLR2, and HMGA2 & $\square[137-143]$ \\
\hline miR-497 & +24.62 & 0.044 & $\begin{array}{l}\square \text { Tumor suppressor: targets CCNE1, insulin-like growth factor } 1 \text { receptor (IGF-1R), } \\
\text { checkpoint kinase } 1 \text { (CHK1), represses elF4E, E3 ubiquitin ligase }\end{array}$ & $\square[89-92,144]$ \\
\hline miR-17-5p & +40.84 & 0.029 & $\begin{array}{l}\square \text { Cellular proliferation: targets SMAD7, FBXO31, E2F1, and c-Myc } \\
\square \text { Repressed HCV production, inversely correlated with HCV treatment response } \\
\square \text { HIV-1 Tat C modulates NOX2 and NOX4 via miR-17-5p } \\
\square \text { Immune modulatory: suppresses TLR signaling through IL-6 and represses } \\
\text { IFN-stimulated MXA expression }\end{array}$ & $\square[121,122]$ \\
\hline miR-205 & +41.22 & 0.027 & $\begin{array}{l}\square \text { De-regulates lipid metabolism by targeting ACSL1 } \\
\square \text { Cellular proliferation: targets ZEB1, ZEB2, UbC13, PTEN, IL13RA2, COL5A2, ADM, } \\
\text { CXCR2, XPO6, SPSB1, FMO5, and PSMF1 } \\
\square \text { HBV X protein hyper-methylates promoter of miR-205 to suppress apoptosis }\end{array}$ & $\square[145,146]$ \\
\hline miR-31 & +43.37 & 0.018 & $\begin{array}{l}\square \text { Cellular proliferation: up-regulated in tumor cells } \\
\square \text { Downstream target genes of miR-31 have an effect on lipid metabolism and } \\
\text { adipogenesis } \\
\square \text { Innate immunity related: targets MyD88 and interferes with TLR2 and TLR4 } \\
\text { signaling }\end{array}$ & $\square[147-151]$ \\
\hline \multicolumn{5}{|c|}{ Convalescent Cattle (Non-Carriers) } \\
\hline miR-455-3p & +68.17 & 0.039 & $\square$ Tumor suppressor: targets RAF & $\square[61]$ \\
\hline miR-150 & -1.65 & 0.044 & 口 Immune modulatory: targets MyD88 (key TLR regulator) and CXCR4 & $\square[147]$ \\
\hline
\end{tabular}

List of bovine miRNAs that were observed to be up-regulated and down-regulated in serum samples collected from cattle persistently infected with FMDV ("FMDV carriers") and convalescent cattle that had successfully cleared infection ("non-carriers"). Differential miRNA expression is presented relative to uninfected animals. Shown are the miRNA species, the fold-change in expression levels (in ascending order), and the ascribed function from literature searches of cattle miRNAs and homologous miRNAs from other species with little to no sequence divergence. The miRNAs listed showed a fold-change in expression levels of greater than 1.50 , and had $p$-values of $<0.05$

miRNAs clustered with them. As shown in Fig. 4, 11 of the miRNAs that were observed to be differentially regulated in the serum from cattle during FMDV infection were not clustered with other miRNAs in the Bos taurus genome. The non-clustered miRNAs included: let-7 g, bta-miR-26b, bta-miR-150, bta-miR-34a, bta-miR146a, bta-miR-147, bta-miR-205, bta-miR-455-3p, btamiR-1224, bta-miR-1281, and bta-miR-31. The remaining 8 miRNAs (bta-miR-497, bta-miR-144, bta-miR-181b, bta-
miR-22-5p, bta-miR-23b-5p, bta-miR-17-5p, bta-miR154a, and bta-miR-369-3p) detected in this study were found to be clustered. Of these 8, bta-miR-154a and bta-miR-369-3p were the most heavily clustered miRNAs, which is why a more stringent cluster distance was imposed of $<3,000$ bp apart. These two miRNAs were also the only two from this study that were clustered with each other. Similar to the genomic localization of the detected miRNAs, we 


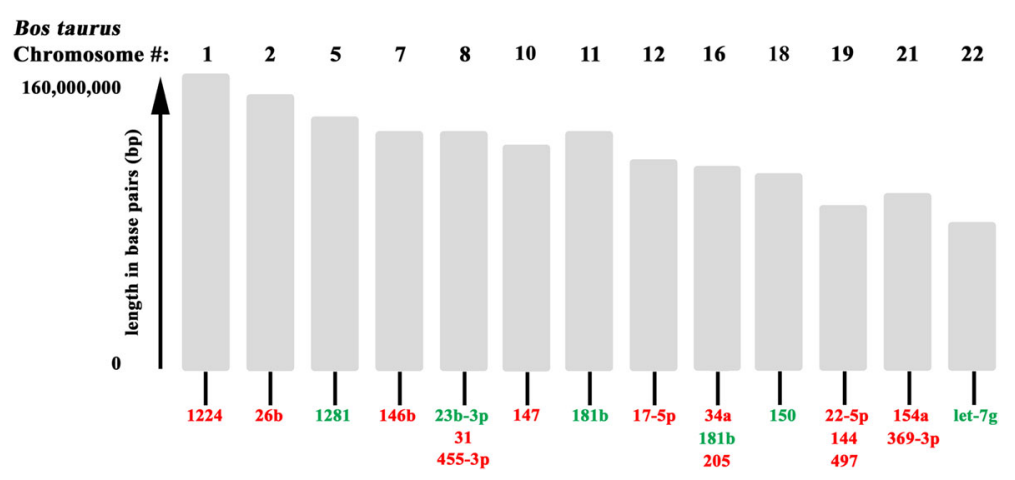

Fig. 3 Genomic localization of miRNAs differentially expressed in response to FMDV infection. The genomic localization of the bovine miRNAs that were found to be differentially expressed in this study were examined on miRbase (current version) for which chromosomes they localized to in the Bos taurus genome. Bovine chromosomes on which miRNA sequences localized were arrayed numerically with the chromosome size in base pairs displayed. miRNAs were listed by their numerical designation in red if down-regulated and in green if up-regulated

concluded that there was no pattern in the clustering data.

\section{Effect of miR-17-5p and miR-1281 on the progression of FMDV infection in vitro}

At the completion of the miRNA profiling analysis, we sought to evaluate the individual impact of one of the up-regulated and one of the down-regulated miRNA species identified on FMDV infection in a cell culture system. Of the miRNA species detected, miR-17-5p and miR-1281 were selected as they were among the 5 miRNA that showed similar differential expression in two different animal sets: acutely infected cattle (Table 2 and Fig. 2) and persistently infected cattle (Table 3 and

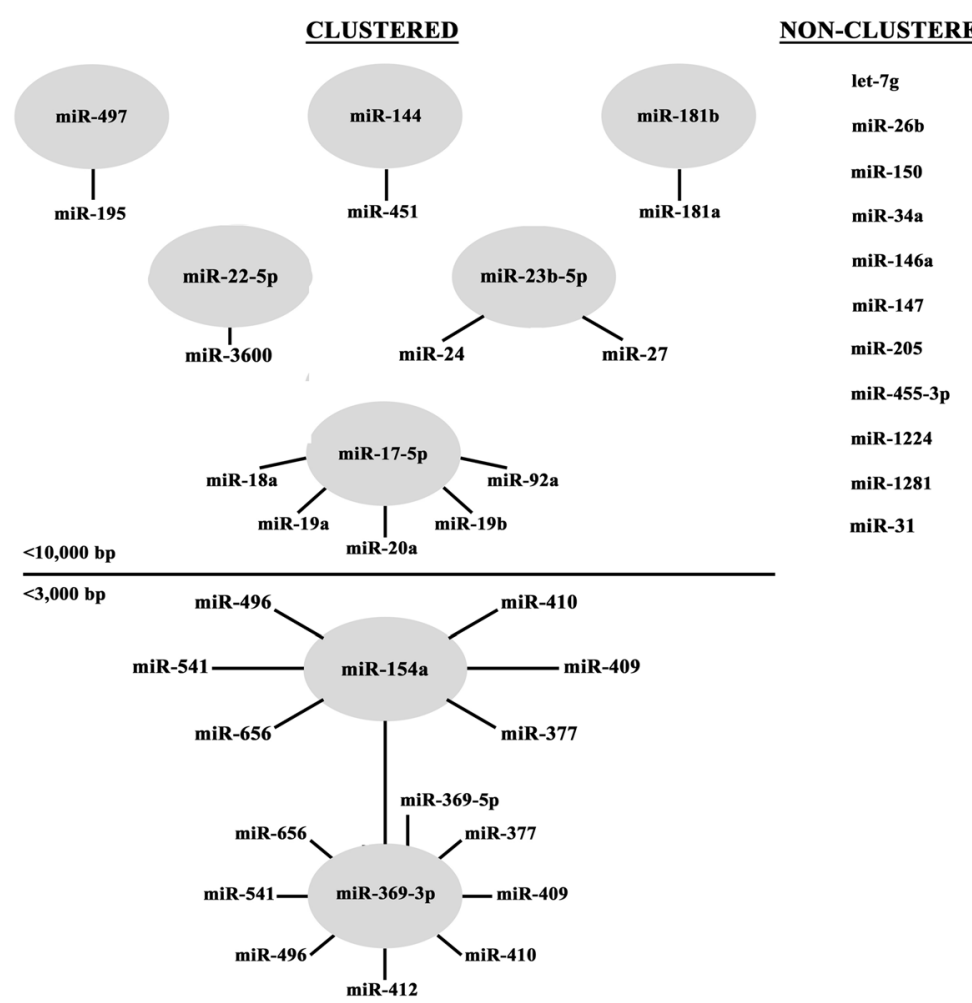

Fig. 4 Cluster analysis of miRNAs differentially expressed in response to FMDV infection. Two different databases were employed to assess whether the differentially expressed miRNAs detected in this study were clustered or non-clustered: miRbase (current version) and MetaMirClust. The miRNAs found to be non-clustered were listed to the right. The miRNAs determined to be clustered were shown in circles with clustered miRNA species indicated around the circle. With the exception of bta-miR-369-3p with a cutoff of $<3000 \mathrm{bp}$, the distance cutoff between miRNA sequences for the cluster analysis was set at $<10,000 \mathrm{bp}$ 
Fig. 2). To that end, mimics of miR-17-5p were transfected into LFBK- $\alpha v \beta 6$ cells, a cell line commonly used for FMDV propagation [37, 38]. Two days posttransfection, cells were infected with FMDV strain A24 Cruzeiro (the same FMDV strain that was used to infect cattle in the current study). It was hypothesized that the elevated expression observed for miR-17-5p in serum samples collected from acutely as well as persistently infected cattle could indicate that this miRNA was either induced by FMDV infection to benefit virus replication, or, induced as a consequence of the host response aiming to suppress virus infection. In the in vitro experiment, there was no impairment to virus replication in cells transfected with miR-17-5p prior to FMDV infection, and virus titers produced were nearly identical to that of the negative controls (untransfected cells and cells transfected non-sense miRNA mimics [miR-NC]) used in the experiment (Fig. 5a).
In a separate experiment, we investigated the potential impact of one of the down-regulated miRNAs that was common to both acutely and persistently infected cattle, miR-1281, on the progression of FMDV infection in vitro. As described for miR-17-5p above, mimic molecules of miR-1281 were transfected into LFBK- $\alpha v \beta 6$ cells and 2 days post-transfection, the cells were infected with FMDV A24 Cruzeiro. In contrast to the findings with miR-17-5p, the transfection of miR-1281 prior to FMDV infection had a negative impact on the progression of FMDV infection confirmed by a 2 to $3 \log$ reduction in viral titers observed in transfected cells compared to controls (Fig. 5b). These in vitro findings are consistent with the results of the in vivo miRNA-profiling study, in that an invading virus (in this case FMDV) would likely attempt to induce the down-regulation of miRNAs that would be detrimental to its replication cycle, while upregulating those that would be beneficial.

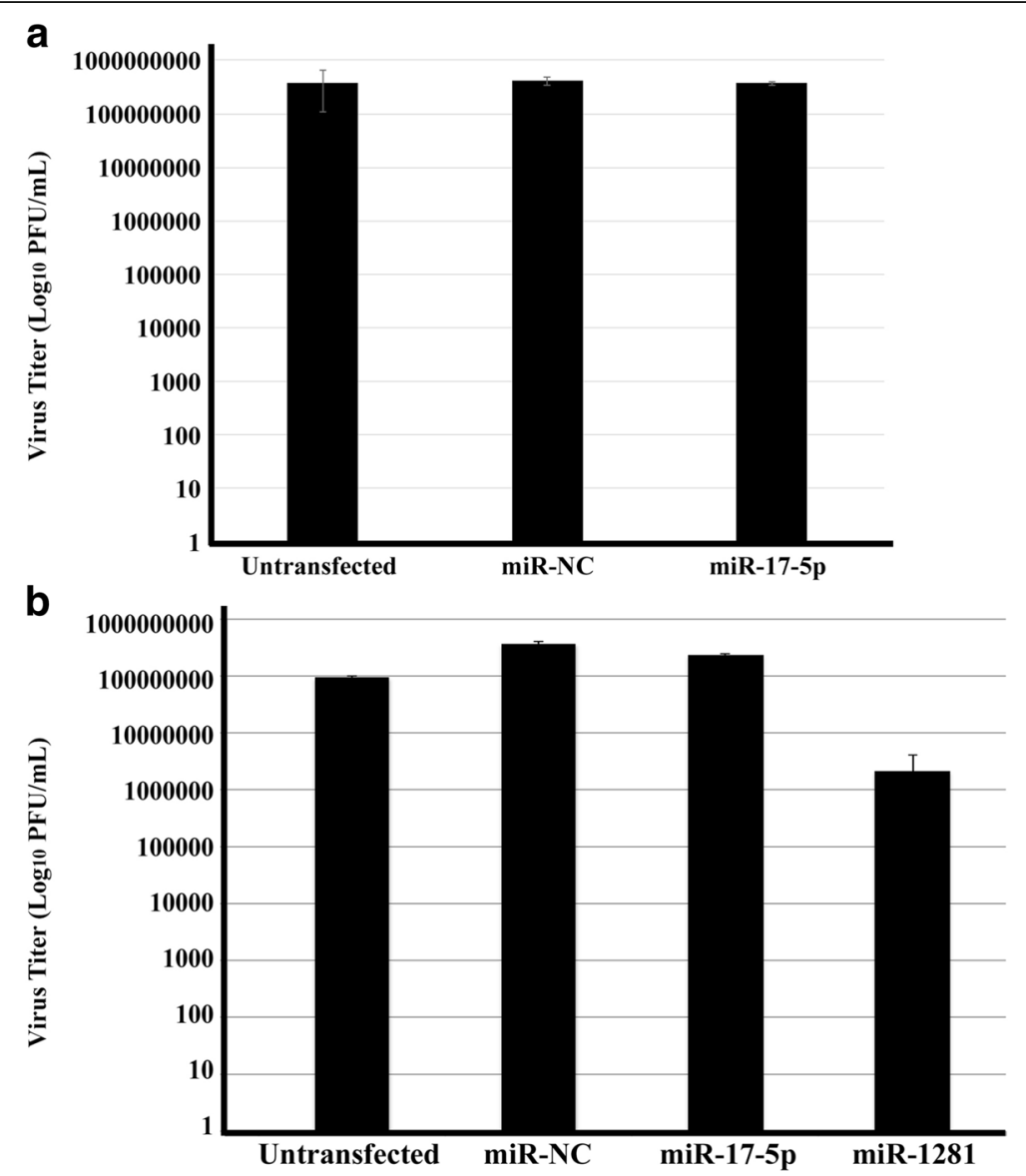

Fig. 5 Effect of miR-17-5p and miR-1281 mimics on FMDV infection in cell culture. a Cells were transfected with miR-17-5p mimics in parallel with negative controls: untransfected and irrelevant miR-342-5p. Forty-eight hours post-transfection, the cells were infected with FMDV A24 Cruzeiro. Subsequent to infection, the resulting samples were evaluated for virus titers, which are plotted. b Cells were transfected with miR-1281 mimics following the same procedure as miR-17-5p (a). Resulting virus titers are plotted as shown. Both panels $\mathbf{a}$ and $\mathbf{b}$ are representative of two independent experiments that were performed in duplicate. miR-17-5p was included in the second experimental set in panel $\mathbf{b}$ to confirm the non-effect seen in panel a 


\section{Discussion}

In viral disease research, miRNA profiling has become a topic of considerable interest as researchers search for cellular targets that can be exploited in order to combat infection. In the case of FMDV, the sole miRNA profiling study to date was an investigation of the differential expression of miRNAs in a porcine based cell line in response to infection with an isolate of FMDV serotype Asia1 [49]. The widespread utility of these findings is somewhat limited given that cell culture is not necessarily reflective of the molecular pathogenesis of the virus in an in vivo setting. miRNA profiling analysis of tissue, urine, and serum samples from infected organisms is being conducted with greater frequency. Using miRNA RT-qPCR profiling arrays containing 169 characterized miRNAs found in Bos taurus, we investigated the differential levels of miRNAs in sera collected from cattle during three distinct phases of infection with FMDV serotype A isolate A24 Cruzeiro, including acute and persistent phases of infection. Moreover, persistent phase samples were obtained from sub-clinically infected FMDV carriers as well as convalescent animals that had successfully cleared the infection. A total of 19 miRNAs exhibited an altered abundance in serum in response to FMDV infection. Five of the miRNAs identified with significant shifts in circulating levels relative to uninfected animals were unique to acutely infected cattle (Fig. 6a, orange circle), seven were only observed in serum from persistently infected carriers (Fig. 6a, blue circle), whereas two were specific to convalescent animals (Fig. 6a, green circle). Of the miRNAs that were significantly up- or down-regulated, five were shared between acutely and persistently infected cattle (bta-miR-17-5p, bta-miR-144, bta-miR-497, bta-miR-22-5p, and bta-miR1281). Mimics of miR-17-5p and miR-1281 were separately tested for their effect on FMDV replication in cell culture (Fig. 5). While miR-17-5p had no apparent impact on the progression of FMDV infection, mimics of
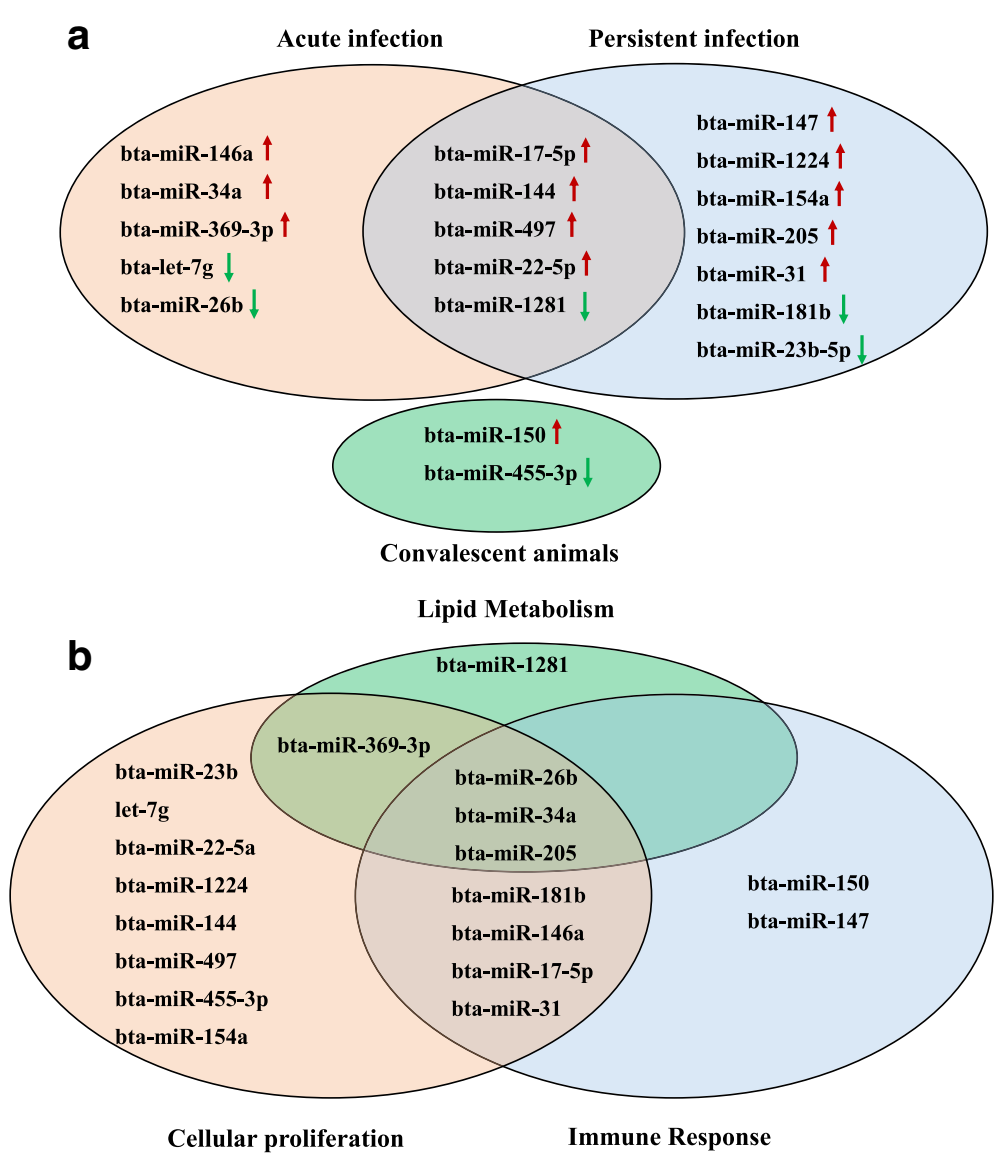

Fig. 6 Schematic overview of the general function of the miRNAs found to be differentially expressed in cattle serum in response to FMDV infection. a The miRNAs identified in the study that are unique to acute infection (orange circle), persistent infection (blue circle), and convalescent animals (green circle) are shown, as well as those shared between acutely and persistently infected cattle. Red and green arrows indicate if targets were up- or downregulated. $\mathbf{b}$. The functionality of the miRNAs detected could be separated into three general categories: cellular proliferation (orange circle), immune response (blue circle), and lipid metabolism/adipogenesis (green circle). A Venn diagram was constructed showing which miRNAs exhibited functionality exclusive to one category and which miRNAs overlapped into more than one of the aforementioned categories 
miR-1281 decreased the resulting FMDV titers by $2-3$ logs. From these findings, it could be inferred that the induction of miR-17-5p in vivo might manipulate the cellular environment such that it favors FMDV replication, while miR-1281 is down-regulated due to an antagonistic effect. Given that miR-1281 mimics impede FMDV replication and the reported role of miR-1281 in cattle [50], it seems that the state of lipid homeostasis in the host plays an important role in the outcome of FMDV infection regardless of whether it is acute or persistent. A survey of all the predicted target genes of these two miRNAs using multiple online algorithms failed to produce a consensus target to be explored in this current study (see Additional file 2: Table S1).

The previously reported miRNA profile of a porcine cell line infected with FMDV serotype Asia1 was obtained using high throughput sequencing [49], which represents the first time such a differential profiling experiment was performed for FMDV. The present study represents the first miRNA profiling experiment, albeit a limited small scale proof-of-concept study, using serum collected from uninfected and infected cattle. This study also marks the first time this type of analysis was performed for a serotype A FMDV isolate (A24 Cruzeiro) as well as the first time differentially expressed miRNAs were examined in the context of persistent FMDV infection. Four of the miRNAs detected were shared between the two profiling studies: miR-22-5p, miR-146b, miR23b-5p, and miR-369-3p. Notably, these four miRNAs all participate in the regulation of cell proliferation and apoptosis. These may also potentially represent four miRNAs that are distinctive to FMDV infection across all serotypes, while the targets not shared between these two studies represent sets of miRNAs that are either specific to the FMDV strain or the experimental system (cell culture versus infected cattle). Future profiling studies with other FMDV serotypes will be necessary to bring certainty to these suppositions. We also wanted to examine the differential miRNA profiles in serum from cattle infected with other viral pathogens; however, we were unable to find published reports comparable to the proof-of-concept study described herein. Despite that, the miRNA profiles generated from human serum collected from individuals infected with related + ssRNA viruses (Dengue virus and $\mathrm{HCV}$ ) showed some dysregulated miRNAs shared with the ones reported for FMDV here, which included: let-7 g, miR-22-5p, miR-23b-5p, miR-146a, and miR-497 [51, 52]. Of note, these shared miRNAs all are reportedly involved in the signaling pathways involved in cellular proliferation and apoptosis, from which it can be inferred that these pathways are important to life cycle of + ssRNA viruses. Potentially, these shared miRNAs that are differentially regulated in the serum in response to invasion by + ssRNA viruses suggest that common molecular mechanisms are activated or suppressed to either combat + ssRNA viruses or to alter the host environment such to facilitate + ssRNA virus replication.

There is increased evidence to support the value of the application of profiling of miRNAs detectable in circulation as biomarkers suggesting the presence and even prognosis of specific disease conditions. For example, hand-foot-and-mouth disease (HFMD) results from infection by one of many possible Picornaviruses, and research has demonstrated that each of these viruses elicits a unique systemic miRNA response that enables differentiation of the potential causative agents [17]. Thus, it would be of interest to elucidate the signature miRNA response for FMDV infection, which may be applicable across all serotypes of FMDV or could be different with each of the seven known serotypes and in different host organisms such as goats, sheep, and pigs. Similarly, identification of distinct changes in circulating miRNAs potentially associated with subclinical persistence of FMDV could be of great value for development of novel diagnostic approaches for disease surveillance. Furthermore, some of the miRNAs detected in this study might also serendipitously target regions of the FMDV genome, and could be potentially adapted as biotherapeutic against FMDV similar to recent report with miR-203a-3p and miR-203a-5p [53]. To briefly explore that possibility, a bioinformatics analysis was conducted using two miRNA recognition sequence mapping algorithms (miRmap and ViTa) $[54,55]$, where the sequence for the FMDV isolate used in this study was input (A24 Cruzeiro, Accession \#AY593768) along with each individual miRNA identified herein (see Additional file 3: Table S2). The miRmap algorithm reported that 6 of the 19 miRNAs dysregulated in bovine serum in response to FMDV could potentially target different regions of the FMDV A24 Cruzeiro RNA genome: bta-miR-17-5p, btamiR-497, bta-miR-146a, bta-miR-1224, bta-miR-31, and bta-miR-150. In contrast, the ViTa algorithm found that more of these miRNAs could potentially target the genome, adding bta-miR-205, bta-miR-26b, bta-let-7 g, btamiR-34a, bta-miR-144, bta-miR-181b, and bta-miR-147 to the list. Future studies with these new miRNA targets will certainly need to explore the relative stability of the FMDV genome in the presence of elevated levels of these select miRNAs.

While approximately $87 \%$ of the profiled miRNAs exhibited no change in circulation levels between uninfected and infected animals, roughly $13 \%$ were significantly up-regulated or down-regulated as a result of FMDV infection. Of the differentially regulated miRNAs, 16 (bta-miR-23b-5p, let-7 g, bta-miR-22-5p, bta-miR1224, bta-miR-144, bta-miR-497, bta-miR-455-3p, btamiR-154a, bta-miR-369-3p, bta-miR-26b, bta-miR-34a, 
bta-miR-205, bta-miR-181b, bta-miR-146a, bta-miR-17$5 \mathrm{p}$, and bta-miR-31) have previously been described to play a role in cellular proliferation or apoptosis (Fig. 6b, orange circle). Nine of the miRNAs (bta-miR-26b, btamiR-34a, bta-miR-205, bta-miR-181b, bta-miR-146a, bta-miR-17-5p, bta-miR-31, bta-miR-150, and bta-miR147), have been ascribed immune modulatory functions (Fig. 6b, blue circle). Also notable is that five of the detected miRNAs (bta-miR-1281, bta-miR-369-3p, btamiR-26b, bta-miR-34a, and bta-miR-205) are involved in adipogenesis or other lipid metabolic pathways (Fig. 6b, green circle). Interestingly, there were an equivalent number of immune modulatory miRNAs differentially expressed between acutely and persistently infected cattle with one down-regulated and three up-regulated. Only one of the immune modulatory miRNAs was shared between the two sets: bta-miR-17-5p, which was significantly upregulated during both acute and persistent FMDV infection. This miRNA has been extensively studied for its roles in immune modulation as well as cellular proliferation and apoptosis [56]. Additionally, miR-17-5p has been implicated in T-cell activation, Bcell and monocyte maturation as well as with suppression of TLR signaling and hampering of the IFN response $[16,57,58]$, which are important functions of the host anti-viral response. These two test groups also exhibited approximately the same number of miRNAs associated with cell proliferation and apoptosis with eight and ten differentially expressed in acutely and persistently infected animals, respectively. Interestingly, there were two times more lipid metabolism-related miRNAs with dysregulated expression in acutely infected cattle, suggesting that modulation of such pathways is important to active FMDV replication. It is known that the VP4 structural protein is acylated with myristate [59]; and more recently, a SILAC screening showed that LYPLA, which is a known depalmitoylase, is a critical factor in FMDV infection [60]. It would be expected that the serum miRNAs of an animal that cleared FMDV would return to a basal level similar to an uninfected animal, and consistent with that, the samples obtained from convalescent cattle exhibited only two dysregulated miRNAs: one that is immune modulatory (bta-miR-150) and one that promotes cellular proliferation (bta-miR455-3p). In serum from convalescent animals, bta-miR150 was marginally down-regulated, possibly indicating a reduction in immune system activation given that the FMDV infection was cleared. In strong contrast, btamiR-455-3p was the most up-regulated miRNA in all of the data sets examined in this study. A survey of published findings on miR-455-3p point toward it being a potent upstream regulator of multiple signaling pathways associated with induced cellular proliferation [61-63]. Since one of the cellular mechanism for combating a viral infection is the induction of apoptosis in infected cells, thereby also increasing cellular turnover, the augmentation in bta-miR-455-3p expression may be reflective of the animal reversing those effects and shutting down those pathways now that FMDV infection has been eliminated. As with the bioinformatics analysis conducted to identify prospective gene targets for miR-17-5p and miR-1281, a consensus target gene for miR-455-3p was not found to further investigate (Additional file 2: Table S1). However, consistent with the speculation above, many of the target genes proposed for miR-455-3p were cell cycle regulators and zinc-finger containing proteins, from which it can be inferred that this single miRNA was regulating multiple cell survival pathways to facilitate the final stages of clearing FMDV infection.

It is interesting to note that six of the 19 miRNAs described in this study are considerably abundant in cattle liver: bta-miR-22-5p, bta-miR-150, bta-miR-17-5p, btamiR-455-3p, bta-miR-146, and let 7-g [64]; an organ in which FMDV does not establish infection. However, due to the substantial blood flow through the liver, large quantities of FMDV particles will pass through the liver during high titer viremia. It is unclear if or how FMDV may be affecting this tissue to possibly stimulate the secretion of the aforementioned miRNAs into the circulation. However, acute FMDV infection in cattle has previously been associated with a strong acute phase response characterized by significant induction of serum amyloid A and haptoglobin levels in serum [65]. These proteins are secreted from the liver in response to a systemic inflammatory reaction [66], which further suggests that the systemic effect of FMDV infection may affect hepatic gene expression and protein synthesis. Further investigation will be required to elucidate the extent and potential application of these findings.

In conclusion, we have identified several miRNAs that are elevated or diminished in circulation of cattle that have been infected with FMDV. Interestingly, unique miRNA serum signatures were detected for acutely infected and persistently infected cattle. The unique miRNAs found in serum from persistently infected cattle may help determine what cellular pathways are actively contributing to the development and maintenance of the "FMDV carrier state". Characterization of signature serum miRNAs that are distinct to the FMDV carrier state could facilitate identification of persistently infected animals in post-outbreak surveillance. Moreover, the miRNAs described may also be adapted as potential anti-viral therapeutics as has been attempted with siRNAs in the past $[6-9,67,68]$. Further investigations including increased sample numbers will be needed to verify the findings of the current proof-of-concept study. Additional in vitro investigations may provide further 
knowledge of distinct biological functions and potential therapeutic applications of the bovine miRNAs identified herein.

\section{Conclusions}

Perturbations in the circulating levels of miRNAs in the bloodstream of humans and animals in response to infection can potentially provide diagnostic and prognostic value. Here, for the first time in a small scale proof-ofconcept study, differentially regulated serum miRNAs from cattle infected with FMDV at different phases of infection were reported. Signature circulating miRNAs were identified for acutely and persistently infected animals, which could be applied to the development of diagnostic assays allowing for distinction between carrier and non-carrier cattle.

\section{Additional files}

Additional file 1: Table S3. Cattle serum samples used for the miRNA profiling study. (DOCX $12 \mathrm{~kb}$ )

Additional file 2: Table S1. Predicted mRNA targets for indicated miRNAs. (DOCX $21 \mathrm{~kb}$ )

Additional file 3: Table S2. Predicted FMDV genomic targets for indicated miRNAs. (DOCX $15 \mathrm{~kb}$ )

\section{Abbreviations}

FMDV: Foot-and-mouth disease virus; miRNA, miR: microRNA

\section{Acknowledgments}

The authors of this manuscript wish to thank Dr. Luis Rodriguez for fruitful discussions during the execution of this study and the preparation of this manuscript. The authors also wish to thank Mr. Isaiah Negron for his review of this manuscript and suggested modifications.

\section{Authors' contributions}

CS: collected the cattle serum, participated in the purification of miRNA from the samples and the downstream analysis, and co-wrote the manuscript with the principal investigator (PL). JA: infected the cattle with FMDV A24 Cruzeiro, assisted in the collection of serum samples, contributed to the conception of the study. GS: performed the bulk of the miRNA array plate analysis. ML: assisted in the miRNA mimic study in cell culture. JG: conducted the bulk of the miRNA mimic study in cell culture. $\mathrm{PL}$ : assisted in the purification and downstream analysis of miRNAs harvested from cattle serum, assisted in the miRNA mimic study in cell culture, conceived and designed the overall study, and co-wrote the manuscript with CS. All authors read and approved the final manuscript.

\section{Competing interests}

The authors declare that they have no competing interests.

\section{Ethics approval and consent to participate}

The experimental study performed on the cattle described in this manuscript was conducted following the strictest guidelines of the Institutional Animal Care and Use Committee (IACUC).

\section{Publisher's Note}

Springer Nature remains neutral with regard to jurisdictional claims in published maps and institutional affiliations.
Received: 22 January 2017 Accepted: 29 March 2017

Published online: 07 April 2017

\section{References}

1. Mason PW, Chinsangaram J, Moraes MP, Mayr GA, Grubman MJ. Engineering better vaccines for foot-and-mouth disease. Dev Biol (Basel). 2003;114:79-88.

2. Mason PW, Grubman MJ, Baxt B. Molecular basis of pathogenesis of FMDV. Virus Res. 2003:91:9-32.

3. Cejka D, Losert D, Wacheck V. Short interfering RNA (siRNA): tool or therapeutic? Clin Sci (Lond). 2006:110:47-58.

4. Karagiannis TC, El-Osta A. RNA interference and potential therapeutic applications of short interfering RNAs. Cancer Gene Ther. 2005;12:787-95.

5. Spurgers KB, Sharkey CM, Warfield KL, Bavari S. Oligonucleotide antiviral therapeutics: antisense and RNA interference for highly pathogenic RNA viruses. Antiviral Res. 2008;78:26-36.

6. Chen W, Liu M, Jiao Y, Yan W, Wei X, Chen J, Fei L, Liu Y, Zuo X, Yang F, et al. Adenovirus-mediated RNA interference against foot-and-mouth disease virus infection both in vitro and in vivo. J Virol. 2006;80:3559-66.

7. de los Santos T, Wu Q, de Avila Botton S, Grubman MJ. Short hairpin RNA targeted to the highly conserved $2 \mathrm{~B}$ nonstructural protein coding region inhibits replication of multiple serotypes of foot-and-mouth disease virus. Virology. 2005;335:222-31.

8. Grubman MJ, de los Santos T. Rapid control of foot-and-mouth disease outbreaks: is RNAi a possible solution? Trends Immunol. 2005;26:65-8.

9. Liu M, Chen W, Ni Z, Yan W, Fei L, Jiao Y, Zhang J, Du Q, Wei X, Chen J, et al. Cross-inhibition to heterologous foot-and-mouth disease virus infection induced by RNA interference targeting the conserved regions of viral genome. Virology. 2005;336:51-9.

10. Carroll AP, Goodall GJ, Liu B. Understanding principles of miRNA target recognition and function through integrated biological and bioinformatics approaches. Wiley Interdiscip Rev RNA. 2014;5:361-79.

11. Dalmay T. Mechanism of miRNA-mediated repression of mRNA translation. Essays Biochem. 2013;54:29-38

12. Lam JK, Chow MY, Zhang Y, Leung SW. siRNA versus miRNA as therapeutics for gene silencing. Mol Ther Nucleic Acids. 2015;4:e252.

13. Thomas M, Lieberman J, Lal A. Desperately seeking microRNA targets. Nat Struct Mol Biol. 2010;17:1169-74.

14. Bueno MJ, Perez de Castro I, Malumbres M. Control of cell proliferation pathways by microRNAs. Cell Cycle. 2008;7:3143-8.

15. Carleton M, Cleary MA, Linsley PS. MicroRNAs and cell cycle regulation. Cell Cycle. 2007:6:2127-32.

16. Dai R, Ahmed SA. MicroRNA, a new paradigm for understanding immunoregulation, inflammation, and autoimmune diseases. Transl Res. 2011;157:163-79.

17. Cui L, Qi Y, Li H, Ge Y, Zhao K, Qi X, Guo X, Shi Z, Zhou M, Zhu B, et al. Serum microRNA expression profile distinguishes enterovirus 71 and coxsackievirus 16 infections in patients with hand-foot-and-mouth disease. PLoS One. 2011;6:e27071.

18. Duy J, Koehler JW, Honko AN, Schoepp RJ, Wauquier N, Gonzalez JP, Pitt ML, Mucker EM, Johnson JC, O'Hearn A, et al. Circulating microRNA profiles of Ebola virus infection. Sci Rep. 2016;6:24496.

19. Hung $\mathrm{CH}, \mathrm{Hu} \mathrm{TH}$, Lu SN, Kuo FY, Chen CH, Wang JH, Huang CM, Lee CM, Lin $C Y$, Yen YH, Chiu YC. Circulating microRNAs as biomarkers for diagnosis of early hepatocellular carcinoma associated with hepatitis B virus. Int J Cancer. 2016;138:714-20.

20. Lee $\mathrm{CH}, \mathrm{Kim} J \mathrm{H}$, Lee SW. The role of MicroRNA in pathogenesis and as markers of HCV chronic infection. Curr Drug Targets. 2016;17:1-10.

21. Wang C, Hann HW, Ye Z, Hann RS, Wan S, Ye X, Block PD, Li B, Myers RE, Wang $X$, et al. Prospective evidence of a circulating microRNA signature as a non-invasive marker of hepatocellular carcinoma in HBV patients. Oncotarget. 2016;9429:1-12.

22. Lawless N, Vegh P, O'Farrelly C, Lynn DJ. The role of microRNAs in Bovine infection and immunity. Front Immunol. 2014;5:611.

23. Kelly EJ, Hadac EM, Cullen BR, Russell SJ. MicroRNA antagonism of the picornaviral life cycle: alternative mechanisms of interference. PLoS Pathog. 2010;6:e1000820.

24. Yen LC, Lin YL, Sung HH, Liao JT, Tsao CH, Su CM, Lin CK, Liao CL. Neurovirulent flavivirus can be attenuated in mice by incorporation of neuron-specific microRNA recognition elements into viral genome. Vaccine. 2013;31:5915-22. 
25. Feng $C$, Tan M, Sun W, Shi Y, Xing Z. Attenuation of the influenza virus by microRNA response element in vivo and protective efficacy against 2009 pandemic H1N1 virus in mice. Int J Infect Dis. 2015;38:146-52.

26. Perez JT, Pham AM, Lorini MH, Chua MA, Steel J, tenOever BR. MicroRNAmediated species-specific attenuation of influenza A virus. Nat Biotechnol. 2009:27:572-6.

27. Hussain M, Torres S, Schnettler E, Funk A, Grundhoff A, Pijlman GP, Khromykh AA, Asgari S. West Nile virus encodes a microRNA-like small RNA in the $3^{\prime}$ untranslated region which up-regulates GATA4 mRNA and facilitates virus replication in mosquito cells. Nucleic Acids Res. 2012;40: 2210-23.

28. Shi J, Duan Z, Sun J, Wu M, Wang B, Zhang J, Wang H, Hu N, Hu Y. Identification and validation of a novel microRNA-like molecule derived from a cytoplasmic RNA virus antigenome by bioinformatics and experimental approaches. Virol J. 2014;11:121.

29. Shi J, Sun J, Wang B, Wu M, Zhang J, Duan Z, Wang H, Hu N, Hu Y. Novel microRNA-like viral small regulatory RNAs arising during human hepatitis $A$ virus infection. FASEB J. 2014;28:4381-93.

30. Chang Y, Dou Y, Bao H, Luo X, Liu X, Mu K, Liu Z, Liu X, Cai X. Multiple microRNAs targeted to internal ribosome entry site against foot-and-mouth disease virus infection in vitro and in vivo. Virol J. 2014;11:1

31. Du J, Gao S, Luo J, Zhang G, Cong G, Shao J, Lin T, Cai X, Chang H. Effective inhibition of foot-and-mouth disease virus (FMDV) replication in vitro by vector-delivered microRNAs targeting the 3D gene. Virol J. 2011;8:292.

32. Gismondi MI, Ortiz XP, Curra AP, Asurmendi S, Taboga O. Artificial microRNAs as antiviral strategy to FMDV: structural implications of target selection. J Virol Methods. 2014;199:1-10.

33. Stenfeldt C, Eschbaumer M, Pacheco JM, Rekant SI, Rodriguez LL, Arzt J. Pathogenesis of primary foot-and-mouth disease virus infection in the nasopharynx of vaccinated and Non-vaccinated cattle. PLoS One. 2015;10: e0143666.

34. Stenfeldt C, Eschbaumer M, Rekant SI, Pacheco JM, Smoliga GR, Hartwig EJ, Rodriguez LL, Arzt J. The foot-and-mouth disease carrier state divergence in cattle. J Virol. 2016;90:6344-64.

35. Pacheco JM, Stenfeldt C, Rodriguez LL, Arzt J. Infection dynamics of footand-mouth disease virus in cattle following intranasopharyngeal inoculation or contact exposure. J Comp Pathol. 2016;155(4):314-25.

36. Livak KJ, Schmittgen TD. Analysis of relative gene expression data using real-time quantitative PCR and the 2(-Delta Delta C(T)) Method. Methods. 2001;25:402-8.

37. LaRocco M, Krug PW, Kramer E, Ahmed Z, Pacheco JM, Duque H, Baxt B, Rodriguez LL. A continuous bovine kidney cell line constitutively expressing bovine alphavbeta6 integrin has increased susceptibility to foot-and-mouth disease virus. J Clin Microbiol. 2013;51:1714-20.

38. Swaney LM. A continuous bovine kidney cell line for routine assays of footand-mouth disease virus. Vet Microbiol. 1988;18:1-14.

39. Rieder E, Bunch T, Brown F, Mason PW. Genetically engineered foot-andmouth disease viruses with poly $(C)$ tracts of two nucleotides are virulent in mice. J Virol. 1993;67:5139-45.

40. Pacheco JM, Stenfeldt C, Rodriguez LL, Arzt J. Infection dynamics of footand-mouth disease virus in cattle following intranasopharyngeal inoculation or contact exposure. J Comp Pathol. 2016;155:314-25.

41. Alexandersen S, Zhang Z, Donaldson Al. Aspects of the persistence of footand-mouth disease virus in animals-the carrier problem. Microbes Infect. 2002:4:1099-110.

42. Salt JS. The carrier state in foot and mouth disease-an immunological review. Br Vet J. 1993;149:207-23.

43. Burrows R. Studies on the carrier state of cattle exposed to foot-and-mouth disease virus. J Hyg (Lond). 1966;64:81-90.

44. Pacheco JM, Smoliga GR, O'Donnell V, Brito BP, Stenfeldt C, Rodriguez LL, Arzt J. Persistent foot-and-mouth disease virus infection in the nasopharynx of cattle; tissue-specific distribution and local cytokine expression. PLoS One. 2015;10:e0125698.

45. Eschbaumer M, Stenfeldt C, Rekant SI, Pacheco JM, Hartwig EJ, Smoliga GR, Kenney MA, Golde WT, Rodriguez LL, Arzt J. Systemic immune response and virus persistence after foot-and-mouth disease virus infection of naive cattle and cattle vaccinated with a homologous adenovirus-vectored vaccine. BMC Vet Res. 2016;12:205.

46. Altuvia $Y$, Landgraf $P$, Lithwick G, Elefant N, Pfeffer S, Aravin A, Brownstein MJ, Tuschl T, Margalit H. Clustering and conservation patterns of human microRNAs. Nucleic Acids Res. 2005;33:2697-706.
47. Yu J, Wang F, Yang GH, Wang FL, Ma YN, Du ZW, Zhang JW. Human microRNA clusters: genomic organization and expression profile in leukemia cell lines. Biochem Biophys Res Commun. 2006;349:59-68.

48. Chan WC, Ho MR, Li SC, Tsai KW, Lai CH, Hsu CN, Lin WC. MetaMirClust: discovery of miRNA cluster patterns using a data-mining approach. Genomics. 2012:100:141-8.

49. Zhang KS, Liu YJ, Kong HJ, Cheng WW, Shang YJ, Tian H, Zheng HX, Guo JH, Liu XT. Identification and analysis of differential miRNAs in PK-15 cells after foot-and-mouth disease virus infection. PLoS One. 2014;9:e90865.

50. Romao JM, Jin W, He M, McAllister T, Guan le L. MicroRNAs in bovine adipogenesis: genomic context, expression and function. BMC Genomics. 2014;15:137.

51. Ouyang X, Jiang X, Gu D, Zhang Y, Kong SK, Jiang C, Xie W. Dysregulated serum MiRNA profile and promising biomarkers in dengue-infected patients. Int J Med Sci. 2016:13:195-205.

52. Zhang S, Ouyang X, Jiang X, Gu D, Lin Y, Kong SK, Xie W. Dysregulated serum MicroRNA expression profile and potential biomarkers in hepatitis $C$ virus-infected patients. Int J Med Sci. 2015;12:590-8.

53. Gutkoska J, LaRocco M, Ramirez-Medina E, de Los Santos T, Lawrence P. Host microRNA-203a Is antagonistic to the progression of foot-and-mouth disease virus infection. Virology. 2017:504:52-62.

54. Hsu PW, Lin LZ, Hsu SD, Hsu JB, Huang HD. ViTa: prediction of host microRNAs targets on viruses. Nucleic Acids Res. 2007;35:D381-5.

55. Vejnar CE, Zdobnov EM. MiRmap: comprehensive prediction of microRNA target repression strength. Nucleic Acids Res. 2012;40:11673-83.

56. Qu Y, Zhang H, Duan J, Liu R, Deng T, Bai M, Huang D, Li H, Ning T, Zhang $L$, et al. MiR-17-5p regulates cell proliferation and migration by targeting transforming growth factor-beta receptor 2 in gastric cancer. Oncotarget. 2016;7:33286-96.

57. Tsubota A, Mogushi K, Aizaki H, Miyaguchi K, Nagatsuma K, Matsudaira H, Kushida T, Furihata T, Tanaka H, Matsuura T. Involvement of MAP3K8 and miR-17-5p in poor virologic response to interferon-based combination therapy for chronic hepatitis C. PLoS One. 2014;9:e97078.

58. Bomben R, Gobessi S, Dal Bo M, Volinia S, Marconi D, Tissino E, Benedetti D, Zucchetto A, Rossi D, Gaidano G, et al. The miR-17 approximately 92 family regulates the response to Toll-like receptor 9 triggering of CLL cells with unmutated IGHV genes. Leukemia. 2012;26:1584-93.

59. Belsham GJ, Abrams CC, King AM, Roosien J, Vlak JM. Myristoylation of footand-mouth disease virus capsid protein precursors is independent of other viral proteins and occurs in both mammalian and insect cells. J Gen Virol. 1991;72(Pt 3):747-51.

60. Guo HC, Jin Y, Han SC, Sun SQ, Wei YQ, Liu XJ, Feng X, Liu DX, Liu XT. Quantitative proteomic analysis of BHK-21 cells infected with foot-andmouth disease virus serotype Asia 1. PLoS One. 2015;10:e0132384.

61. Chai J, Wang S, Han D, Dong W, Xie C, Guo H. MicroRNA-455 inhibits proliferation and invasion of colorectal cancer by targeting RAF protooncogene serine/threonine-protein kinase. Tumour Biol. 2015:36:1313-21.

62. Kunz M. MicroRNAs in melanoma biology. Adv Exp Med Biol. 2013;774:103-20.

63. Sand M, Skrygan M, Sand D, Georgas D, Hahn SA, Gambichler T, Altmeyer P, Bechara FG. Expression of microRNAs in basal cell carcinoma. Br J Dermatol. 2012:167:847-55.

64. Al-Husseini W, Chen Y, Gondro C, Herd RM, Gibson JP, Arthur PF. Characterization and profiling of liver microRNAs by RNA-sequencing in cattle divergently selected for residual feed intake. Asian-Australas J Anim Sci. 2016:29:1371-82.

65. Stenfeldt C, Heegaard PM, Stockmarr A, Tjornehoj K, Belsham GJ. Analysis of the acute phase responses of serum amyloid a, haptoglobin and type 1 interferon in cattle experimentally infected with foot-and-mouth disease virus serotype O. Vet Res. 2011;42:66.

66. Baumann H, Gauldie J. The acute phase response. Immunol Today. 1994;15: 74-80.

67. Lv K, Guo Y, Zhang Y, Wang K, Li K, Zhu Y, Sun S. Transient inhibition of foot-and-mouth disease virus replication by siRNAs silencing VP1 protein coding region. Res Vet Sci. 2009;86:443-52.

68. Pengyan W, Yan R, Zhiru G, Chuangfu C. Inhibition of foot-and-mouth disease virus replication in vitro and in vivo by small interfering RNA. Virol J. 2008:5:86.

69. Fognani E, Giannini C, Piluso A, Gragnani L, Monti M, Caini P, Ranieri J, Urraro T, Triboli E, Laffi G, Zignego AL. Role of microRNA profile modifications in hepatitis $C$ virus-related mixed cryoglobulinemia. PLoS One. 2013;8:e62965. 
70. Li J, Kong X, Zhang J, Luo Q, Li X, Fang L. MiRNA-26b inhibits proliferation by targeting PTGS2 in breast cancer. Cancer Cell Int. 2013;13:7.

71. Li J, Li X, Kong X, Luo Q, Zhang J, Fang L. MiRNA-26b inhibits cellular proliferation by targeting CDK8 in breast cancer. Int J Clin Exp Med. 2014;7: 558-65.

72. Liu S, Gao L, Wang X, Xing Y. Respiratory syncytial virus infection inhibits TLR4 signaling via up-regulation of miR-26b. Cell Biol Int. 2015;39:1376-83.

73. Shen G, Lin Y, Yang X, Zhang J, Xu Z, Jia H. MicroRNA-26b inhibits epithelial-mesenchymal transition in hepatocellular carcinoma by targeting USP9X. BMC Cancer. 2014;14:393.

74. Song G, Xu G, Ji C, Shi C, Shen Y, Chen L, Zhu L, Yang L, Zhao Y, Guo X. The role of microRNA-26b in human adipocyte differentiation and proliferation. Gene. 2014,533:481-7.

75. Zhao F, Xu G, Zhou Y, Wang L, Xie J, Ren S, Liu S, Zhu Y. MicroRNA-26b inhibits hepatitis $B$ virus transcription and replication by targeting the host factor CHORDC1 protein. J Biol Chem. 2014;289:35029-41.

76. Zhao N, Wang R, Zhou L, Zhu Y, Gong J, Zhuang SM. MicroRNA-26b suppresses the NF-kappaB signaling and enhances the chemosensitivity of hepatocellular carcinoma cells by targeting TAK1 and TAB3. Mol Cancer. 2014;13:35.

77. Romao JM, Jin W, Dodson MV, Hausman GJ, Moore SS, Guan LL. MicroRNA regulation in mammalian adipogenesis. Exp Biol Med (Maywood). 2011;236: 997-1004.

78. Ding Z, Wang X, Khaidakov M, Liu S, Mehta JL. MicroRNA hsa-let-7g targets lectin-like oxidized low-density lipoprotein receptor-1 expression and inhibits apoptosis in human smooth muscle cells. Exp Biol Med (Maywood). 2012;237:1093-100.

79. Liu JM, Long XH, Zhang GM, Zhou Y, Chen XY, Huang SH, Liu ZL, Zhang ZH. Let-7g reverses malignant phenotype of osteosarcoma cells by targeting Aurora-B. Int J Clin Exp Pathol. 2014;7:4596-606.

80. Zhang Y, Chen N, Zhang J, Tong Y. Hsa-let-7g miRNA targets caspase-3 and inhibits the apoptosis induced by ox-LDL in endothelial cells. Int J Mol Sci. 2013;14:22708-20.

81. Chen H, Lu Q, Fei X, Shen L, Jiang D, Dai D. miR-22 inhibits the proliferation, motility, and invasion of human glioblastoma cells by directly targeting SIRT1. Tumour Biol. 2016:37:6761-8

82. Chen J, Wu FX, Luo HL, Liu JJ, Luo T, Bai T, Li LQ, Fan XH. Berberine upregulates miR-22-3p to suppress hepatocellular carcinoma cell proliferation by targeting Sp1. Am J Transl Res. 2016;8:4932-41.

83. Diez-Planelles C, Sanchez-Lozano P, Crespo MC, Gil-Zamorano J, Ribacoba R, Gonzalez N, Suarez E, Martinez-Descals A, Martinez-Camblor P, Alvarez V, et al. Circulating microRNAs in Huntington's disease: emerging mediators in metabolic impairment. Pharmacol Res. 2016;108:102-10.

84. Jiang $X, H u$ C, Arnovitz S, Bugno J, Yu M, Zuo Z, Chen P, Huang H, Ulrich B, Gurbuxani $S$, et al. miR-22 has a potent anti-tumour role with therapeutic potential in acute myeloid leukaemia. Nat Commun. 2016;7:11452.

85. Liu HL, Zhu JG, Liu YQ, Fan ZG, Zhu C, Qian LM. Identification of the microRNA expression profile in the regenerative neonatal mouse heart by deep sequencing. Cell Biochem Biophys. 2014;70:635-42.

86. Maciejak A, Kiliszek M, Opolski G, Segiet A, Matlak K, Dobrzycki S, Tulacz D, Sygitowicz G, Burzynska B, Gora M. miR-22-5p revealed as a potential biomarker involved in the acute phase of myocardial infarction via profiling of circulating microRNAs. Mol Med Rep. 2016;14:2867-75.

87. Wan S, Ashraf U, Ye J, Duan X, Zohaib A, Wang W, Chen Z, Zhu B, Li Y, Chen $\mathrm{H}$, Cao S. MicroRNA-22 negatively regulates poly(l:C)-triggered type I interferon and inflammatory cytokine production via targeting mitochondrial antiviral signaling protein (MAVS). Oncotarget. 2016;7:76667-83.

88. Zhang S, Zhang D, Yi C, Wang Y, Wang H, Wang J. MicroRNA-22 functions as a tumor suppressor by targeting SIRT1 in renal cell carcinoma. Oncol Rep. 2016;35:559-67

89. Li W, Jin X, Deng X, Zhang G, Zhang B, Ma L. The putative tumor suppressor microRNA-497 modulates gastric cancer cell proliferation and invasion by repressing elF4E. Biochem Biophys Res Commun. 2014;449:235-40.

90. Luo M, Shen D, Zhou X, Chen X, Wang W. MicroRNA-497 is a potentia prognostic marker in human cervical cancer and functions as a tumor suppressor by targeting the insulin-like growth factor 1 receptor. Surgery. 2013;153:836-47

91. Wang W, Ren F, Wu Q, Jiang D, Li H, Peng Z, Wang J, Shi H. MicroRNA-497 inhibition of ovarian cancer cell migration and invasion through targeting of SMAD specific E3 ubiquitin protein ligase 1. Biochem Biophys Res Commun. 2014:449:432-7.
92. Xie Y, Wei RR, Huang GL, Zhang MY, Yuan YF, Wang HY. Checkpoint kinase 1 is negatively regulated by miR-497 in hepatocellular carcinoma. Med Oncol. 2014;31:844.

93. Xu J, Wang T, Cao Z, Huang H, Li J, Liu W, Liu S, You L, Zhou L, Zhang T, Zhao Y. MiR-497 downregulation contributes to the malignancy of pancreatic cancer and associates with a poor prognosis. Oncotarget. 2014;5: 6983-93.

94. Bork S, Horn P, Castoldi M, Hellwig I, Ho AD, Wagner W. Adipogenic differentiation of human mesenchymal stromal cells is down-regulated by microRNA-369-5p and up-regulated by microRNA-371. J Cell Physiol. 2011. 226:2226-34.

95. Rago L, Beattie R, Taylor V, Winter J. miR379-410 cluster miRNAs regulate neurogenesis and neuronal migration by fine-tuning $\mathrm{N}$-cadherin. EMBO J. 2014;33:906-20.

96. Izumi H, Kosaka N, Shimizu T, Sekine K, Ochiya T, Takase M. Bovine milk contains microRNA and messenger RNA that are stable under degradative conditions. J Dairy Sci. 2012;95:4831-41.

97. Sun F, Fu H, Liu Q, Tie Y, Zhu J, Xing R, Sun Z, Zheng X. Downregulation of CCND1 and CDK6 by miR-34a induces cell cycle arrest. FEBS Lett. 2008;582: 1564-8

98. Chang TC, Wentzel EA, Kent OA, Ramachandran K, Mullendore M, Lee KH, Feldmann G, Yamakuchi M, Ferlito M, Lowenstein CJ, et al. Transactivation of miR-34a by p53 broadly influences gene expression and promotes apoptosis. Mol Cell. 2007;26:745-52.

99. Lu G, Sun Y, An S, Xin S, Ren X, Zhang D, Wu P, Liao W, Ding Y, Liang L. MicroRNA-34a targets FMNL2 and E2F5 and suppresses the progression of colorectal cancer. Exp Mol Pathol. 2015;99:173-9.

100. Park H, Park H, Pak HJ, Yang DY, Kim YH, Choi WJ, Park SJ, Cho JA, Lee KW. miR-34a inhibits differentiation of human adipose tissue-derived stem cells by regulating cell cycle and senescence induction. Differentiation. 2015;90: 91-100.

101. Xu Y, Zalzala M, Xu J, Li Y, Yin L, Zhang Y. A metabolic stress-inducible miR-34a-HNF4alpha pathway regulates lipid and lipoprotein metabolism. Nat Commun. 2015;6:7466.

102. Cai SD, Chen JS, Xi ZW, Zhang LJ, Niu ML, Gao ZY. MicroRNA144 inhibits migration and proliferation in rectal cancer by downregulating ROCK1. Mol Med Rep. 2015;12:7396-402.

103. Cao T, Li H, Hu Y, Ma D, Cai X. miR-144 suppresses the proliferation and metastasis of hepatocellular carcinoma by targeting E2F3. Tumour Biol. 2014;35:10759-64.

104. Chen S, Li P, Li J, Wang Y, Du Y, Chen X, Zang W, Wang H, Chu H, Zhao G, Zhang G. MiR-144 inhibits proliferation and induces apoptosis and autophagy in lung cancer cells by targeting TIGAR. Cell Physiol Biochem. 2015:35:997-1007

105. Cheng C, Li W, Zhang Z, Yoshimura S, Hao Q, Zhang C, Wang Z. MicroRNA144 is regulated by activator protein-1 (AP-1) and decreases expression of Alzheimer disease-related a disintegrin and metalloprotease 10 (ADAM10). J Biol Chem. 2013;288:13748-61.

106. Lan F, Yu H, Hu M, Xia T, Yue X. miR-144-3p exerts anti-tumor effects in glioblastoma by targeting c-Met. J Neurochem. 2015;135:274-86.

107. Matsushita R, Seki N, Chiyomaru T, Inoguchi S, Ishihara T, Goto Y, Nishikawa R, Mataki H, Tatarano S, Itesako T, et al. Tumour-suppressive microRNA-144$5 p$ directly targets CCNE1/2 as potential prognostic markers in bladder cancer. Br J Cancer. 2015;113:282-9.

108. Pan Y, Zhang J, Fu H, Shen L. miR-144 functions as a tumor suppressor in breast cancer through inhibiting ZEB1/2-mediated epithelial mesenchymal transition process. Onco Targets Ther. 2016;9:6247-55.

109. Wang W, Zhou X, Wei M. MicroRNA-144 suppresses osteosarcoma growth and metastasis by targeting ROCK1 and ROCK2. Oncotarget. 2015;6:10297308.

110. Zhang J, Qin X, Sun Q, Guo H, Wu X, Xie F, Xu Q, Yan M, Liu J, Han Z, Chen W. Transcriptional control of PAX4-regulated miR-144/451 modulates metastasis by suppressing ADAMs expression. Oncogene. 2015:34:3283-95.

111. Zhang LY, Ho-Fun Lee V, Wong AM, Kwong DL, Zhu YH, Dong SS, Kong KL, Chen J, Tsao SW, Guan XY, Fu L. MicroRNA-144 promotes cell proliferation, migration and invasion in nasopharyngeal carcinoma through repression of PTEN. Carcinogenesis. 2013;34:454-63.

112. Huang C, Geng J, Wei X, Zhang R, Jiang S. MiR-144-3p regulates osteogenic differentiation and proliferation of murine mesenchymal stem cells by specifically targeting Smad4. FEBS Lett. 2016;590:795-807. 
113. Lee HM, Kim TS, Jo EK. MiR-146 and miR-125 in the regulation of innate immunity and inflammation. BMB Rep. 2016;49:311-8.

114. Liang G, Malmuthuge N, Guan Y, Ren Y, Griebel PJ, Guan le L. Altered microRNA expression and pre-mRNA splicing events reveal new mechanisms associated with early stage Mycobacterium avium subspecies paratuberculosis infection. Sci Rep. 2016;6:24964.

115. O'Neill LA, Sheedy FJ, McCoy CE. MicroRNAs: the fine-tuners of Toll-like receptor signalling. Nat Rev Immunol. 2011;11:163-75.

116. Quinn SR, O'Neill LA. A trio of microRNAs that control Toll-like receptor signalling. Int Immunol. 2011;23:421-5.

117. Taganov KD, Boldin MP, Chang KJ, Baltimore D. NF-kappaB-dependent induction of microRNA miR-146, an inhibitor targeted to signaling proteins of innate immune responses. Proc Natl Acad Sci U S A. 2006;103:12481-6.

118. Vegh P, Foroushani AB, Magee DA, McCabe MS, Browne JA, Nalpas NC, Conlon KM, Gordon SV, Bradley DG, MacHugh DE, Lynn DJ. Profiling microRNA expression in bovine alveolar macrophages using RNA-seq. Vet Immunol Immunopathol. 2013;155:238-44.

119. Chang YL, Ho BC, Sher S, Yu SL, Yang PC. miR-146a and miR-370 coordinate enterovirus 71-induced cell apoptosis through targeting SOS1 and GADD45beta. Cell Microbiol. 2015;17:802-18.

120. Li JF, Dai XP, Zhang W, Sun SH, Zeng Y, Zhao GY, Kou ZH, Guo Y, Yu H, Du LY, et al. Upregulation of microRNA-146a by hepatitis $B$ virus $X$ protein contributes to hepatitis development by downregulating complement factor H. MBio. 2015;6(2):e02459-14.

121. Jia J, Feng $X, X u$ W, Yang $S$, Zhang Q, Liu X, Feng $Y$, Dai Z. MiR-17-5p modulates osteoblastic differentiation and cell proliferation by targeting SMAD7 in non-traumatic osteonecrosis. Exp Mol Med. 2014;46:e107

122. Yu F, Guo Y, Chen B, Dong P, Zheng J. MicroRNA-17-5p activates hepatic stellate cells through targeting of Smad7. Lab Invest. 2015;95: 781-9.

123. Brauer-Hartmann D, Hartmann JU, Wurm AA, Gerloff D, Katzerke C, Verga Falzacappa MV, Pelicci PG, Muller-Tidow C, Tenen DG, Niederwieser D, Behre G. PML/RARalpha-regulated miR-181a/b cluster targets the tumor suppressor RASSF1A in acute promyelocytic leukemia. Cancer Res. 2015;75: 3411-24.

124. Kaga H, Komatsuda A, Omokawa A, Ito M, Teshima K, Tagawa H, Sawada K, Wakui H. Downregulated expression of miR-155, miR-17, and miR-181b, and upregulated expression of activation-induced cytidine deaminase and interferon-alpha in PBMCs from patients with SLE. Mod Rheumatol. 2015;25: 865-70.

125. Sun X, Icli B, Wara AK, Belkin N, He S, Kobzik L, Hunninghake GM, Vera MP, Registry M, Blackwell TS, et al. MicroRNA-181b regulates NF-kappaB-mediated vascular inflammation. J Clin Invest. 2012;122: 1973-90.

126. Yang $X$, Chen $X$, Bian G, Tu J, Xing Y, Wang Y, Chen Z. Proteolytic processing, deubiquitinase and interferon antagonist activities of Middle East respiratory syndrome coronavirus papain-like protease. J Gen Virol. 2014;95:614-26.

127. Cabrita MA, Vanzyl EJ, Hamill JD, Pan E, Marcellus KA, Tolls VJ, Alonzi RC, Pastic A, Rambo TM, Sayed H, McKay BC. A temperature sensitive variant of p53 drives p53-dependent MicroRNA expression without evidence of widespread post-transcriptional gene silencing. PLoS One. 2016;11: e0148529.

128. Gao P, Tchernyshyov I, Chang TC, Lee YS, Kita K, Ochi T, Zeller KI, De Marzo AM, Van Eyk JE, Mendell JT, Dang CV. c-Myc suppression of miR-23a/b enhances mitochondrial glutaminase expression and glutamine metabolism. Nature. 2009;458:762-5.

129. Liu W, Zabirnyk O, Wang H, Shiao YH, Nickerson ML, Khalil S, Anderson LM, Perantoni AO, Phang JM. miR-23b targets proline oxidase, a novel tumor suppressor protein in renal cancer. Oncogene. 2010;29:4914-24.

130. Tong AW, Fulgham P, Jay C, Chen P, Khalil I, Liu S, Senzer N, Eklund AC, Han J, Nemunaitis J. MicroRNA profile analysis of human prostate cancers. Cancer Gene Ther. 2009;16:206-16.

131. Xiao X, Huang C, Zhao C, Gou X, Senavirathna LK, Hinsdale M, Lloyd P, Liu L. Regulation of myofibroblast differentiation by miR-424 during epithelial-to-mesenchymal transition. Arch Biochem Biophys. 2015;566: 49-57.

132. Zhang H, Hao Y, Yang J, Zhou Y, Li J, Yin S, Sun C, Ma M, Huang Y, Xi JJ. Genome-wide functional screening of miR-23b as a pleiotropic modulator suppressing cancer metastasis. Nat Commun. 2011;2:554.
133. El-Guendy NM, Helwa R, El-Halawany MS, Abdel Rahman Ali S, Tantawy Aly M, Hasan Alieldin N, Fouad SA, Saeid H, Abdel-Wahab AH. The liver MicroRNA expression profiles associated with chronic hepatitis $C$ virus (HCV) genotype-4 infection: a preliminary study. Hepat Mon. 2016;16: e33881.

134. Liu G, Friggeri A, Yang Y, Park YJ, Tsuruta Y, Abraham E. miR-147, a microRNA that is induced upon Toll-like receptor stimulation, regulates murine macrophage inflammatory responses. Proc Natl Acad Sci U S A. 2009;106:15819-24.

135. Sui CJ, Xu F, Shen WF, Dai BH, Lu JJ, Zhang MF, Yang JM. MicroRNA147 suppresses human hepatocellular carcinoma proliferation migration and chemosensitivity by inhibiting HOXC6. Am J Cancer Res. 2016;6: 2787-98.

136. Qian J, Li R, Wang YY, Shi Y, Luan WK, Tao T, Zhang JX, Xu YC, You YP. MiR-1224-5p acts as a tumor suppressor by targeting CREB1 in malignant gliomas. Mol Cell Biochem. 2015;403:33-41.

137. Li J, Hu C, Han L, Liu L, Jing W, Tang W, Tian W, Long J. MiR-154-5p regulates osteogenic differentiation of adipose-derived mesenchymal stem cells under tensile stress through the Wnt/PCP pathway by targeting Wnt11. Bone. 2015;78:130-41.

138. Lin X, Yang Z, Zhang P, Liu Y, Shao G. miR-154 inhibits migration and invasion of human non-small cell lung cancer by targeting ZEB2. Oncol Lett. 2016;12:301-6.

139. Pang $X$, Huang $K$, Zhang Q, Zhang $Y$, Niu J. miR-154 targeting ZEB2 in hepatocellular carcinoma functions as a potential tumor suppressor. Oncol Rep. 2015;34:3272-9.

140. Xu H, Fei D, Zong S, Fan Z. MicroRNA-154 inhibits growth and invasion of breast cancer cells through targeting E2F5. Am J Transl Res. 2016;8: 2620-30.

141. Zhao D, Wang R, Fang J, Ji X, Li J, Chen X, Sun G, Wang Z, Liu W, Wang Y, et al. MiR-154 functions as a tumor suppressor in glioblastoma by targeting Wnt5a. Mol Neurobiol. 2016;1-8.

142. Zheng Y, Zhu C, Ma L, Shao P, Qin C, Li P, Cao Q, Ju X, Cheng G, Zhu Q, et al. miRNA-154-5p inhibits proliferation, migration and invasion by targeting E2F5 in prostate cancer cell lines. Urol Int. 2016;98(1):102-10.

143. Zhou $H$, Zhang M, Yuan H, Zheng W, Meng C, Zhao D. MicroRNA-154 functions as a tumor suppressor in osteosarcoma by targeting Wnt5a. Oncol Rep. 2016;35:1851-8.

144. Xu JW, Wang TX, You L, Zheng LF, Shu H, Zhang TP, Zhao YP. Insulin-like growth factor 1 receptor (IGF-1R) as a target of MiR-497 and plasma IGF-1R levels associated with TNM stage of pancreatic cancer. PLoS One. 2014;9: e92847.

145. Cui M, Wang Y, Sun B, Xiao Z, Ye L, Zhang X. MiR-205 modulates abnormal lipid metabolism of hepatoma cells via targeting acyl-CoA synthetase longchain family member 1 (ACSL1) mRNA. Biochem Biophys Res Commun. 2014:444:270-5.

146. Cui $M$, Xiao Z, Sun B, Wang Y, Zheng M, Ye L, Zhang X. Involvement of cholesterol in hepatitis $B$ virus $X$ protein-induced abnormal lipid metabolism of hepatoma cells via up-regulating miR-205targeted ACSL4. Biochem Biophys Res Commun. 2014;445:651-5.

147. Ghorpade DS, Holla S, Kaveri SV, Bayry J, Patil SA, Balaji KN. Sonic hedgehog-dependent induction of microRNA 31 and microRNA 150 regulates Mycobacterium bovis BCG-driven toll-like receptor 2 signaling. Mol Cell Biol. 2013;33:543-56.

148. Liu X, Sempere LF, Ouyang H, Memoli VA, Andrew AS, Luo Y, Demidenko $E$, Korc M, Shi W, Preis $M$, et al. MicroRNA-31 functions as an oncogenic microRNA in mouse and human lung cancer cells by repressing specific tumor suppressors. J Clin Invest. 2010;120: 1298-309.

149. Naeem A, Zhong K, Moisa SJ, Drackley JK, Moyes KM, Loor JJ. Bioinformatics analysis of microRNA and putative target genes in bovine mammary tissue infected with Streptococcus uberis. I Dairy Sci. 2012;95:6397-408.

150. Satoh M, Takahashi Y, Tabuchi T, Tamada M, Takahashi K, Itoh T, Morino $Y$, Nakamura M. Circulating Toll-like receptor 4-responsive microRNA panel in patients with coronary artery disease: results from prospective and randomized study of treatment with reninangiotensin system blockade. Clin Sci (Lond). 2015;128:483-91.

151. Tang YF, Zhang Y, Li XY, Li C, Tian W, Liu L. Expression of miR-31, miR-125b-5p, and miR-326 in the adipogenic differentiation process of adipose-derived stem cells. OMICS. 2009;13:331-6. 